\title{
Output Units of Motor Behavior: An Experimental and Modeling Study
}

\author{
E. P. Loeb \\ Gore 2000, Nashville, TN, USA
}

\section{S. F. Giszter}

Medical College of Pennsylvania and Hahnemann University

\section{P. Saltiel and E. Bizzi}

Massachusetts Institute of Technology

\section{F. A. Mussa-Ivaldi}

Northwestern University Medical School

\begin{abstract}
Cognitive approaches to motor control typically concern sequences of discrete actions without taking into account the stunning complexity of the geometry and dynamics of the muscles. This begs the question: Does the brain convert the intricate, continuous-time dynamics of the muscles into simpler discrete units of actions, and if so, how? One way for the brain to form discrete units of behavior from muscles is through the synergistic co-activation of muscles. While this possibility has long been known, the composition of potential muscle synergies has remained elusive. In this paper, we have focused on a method that allowed us to examine and compare the limb stabilization properties of all possible muscle combinations. We found that a small set (as few as 23 out of 65,536) of all possible combinations of 16 limb muscles are robust with respect to activation noise: these muscle combina-
\end{abstract}

\section{INTRODUCTION}

There is no doubt that co-activation of muscles is routinely observed during the execution of movements, but it is not yet clear how particular combinations of muscles are selected by the central nervous system (CNS) for use in these movements. No natural movement is executed by just one muscle, and common movements involve many muscles. Some muscles produce torques and displacements about joints, while others apparently act to maintain postures and oppose the reaction torques produced by limb displacement. The concept of a "muscle synergy" is used to express the notion that there is a functional underlying purpose to the groupings of muscles used to execute movements. The use and re-use of muscle groups characterize every movement. The question is, how is a given muscle group specified by the CNS? tions could stabilize the limb at predictable, restricted portions of the workspace in spite of broad variations in the force output of their component muscles. The locations at which the robust synergies stabilize the limb are not uniformly distributed throughout the leg's workspace, but rather, they cluster at four workspace areas. The simulated robust synergies are similar to the actual synergies we have previously found to be generated by activation of the spinal cord. Thus, we have developed a new analytical method that enabled us to select a few muscle synergies with interesting properties out of the set of possible muscle combinations. Beyond this, the identification of robustness as a common property of the synergies in simple motor behaviors will open the way to the study of dynamic stability, which is an important and distinct property of the vertebrate motor-control system.
In the past, investigators have put forward two radically different views. According to one view, each muscle involved in a task is independently specified by the CNS (Soechting \& Lacquaniti, 1989). In this view, apparent muscle synergies are an artifact of the cross-subject commonality of the physics of movements. Alternatively, there are fixed synergies, which are obligatory linkages between muscles. In this later view, the CNS generates movements by recruiting and combining the fixed synergies in a flexible way (MacPherson, 1991).

Some of the early ideas about synergies were proposed by Sherrington (Sherington, 1961), who conceived the muscle synergies as resulting from the activation of specific reflex pathways. Bernstein (1971) also proposed the concept of synergies. He regarded synergies as a way for the CNS to deal with the many 
degrees of freedom that are involved in the generation of movement. ${ }^{1}$ A synergy in Bernstein's terms is a means, or the means, to reduce the number of mechanical variables involved in the execution of even the simplest movement.

In this work, we have investigated muscle synergies from a new perspective; we have taken the point of view that a collection of co-activated muscles is equivalent to a forcing function acting on the limb dynamics. To understand the concept of a forcing function, suspend a series of weights from a rubber band. When the weight changes, the rubber band will stretch and bounce, but it will eventually settle down at a new length determined by the total magnitude of the weight it is bearing. The weight is a forcing function. The rubber band is a dynamic system. The rubber band has a temporal response to the onset of the forcing function-it bounces-but after that, it settles into its new steady state, which is dictated by the forcing function. In a similar manner, the limb should ultimately settle into a steady state dictated by the total forces exerted by gravity, friction, objects in the environment, and the sum total of the forces exerted by the individual muscles. Thus, we have approached the problem of understanding muscle co-activations by examining muscles and combinations of muscles as forcing functions.

The key instrument that has made this exploration possible is the representation of a muscle's action as a force field. Our force fields map limb postures to the force acting on the ankle at that limb posture. A force field is a forcing function. All else being equal, we expect the ankle (and the limb with it) to accelerate in the direction of any force acting on it. If the force field contains a Convergent Equilibrium Point (CEP) where the force is zero, and towards which the other forces point, then we would expect the limb to move to the position of the CEP and stay there.

In order to predict the limb stabilization properties of combinations of muscles, we have measured the force fields produced by individual muscles, and then combined those force fields. The bulk of this study concerns the validation of the method. We first developed and tested several different models of individual muscles. We then performed statistical tests to verify that the modeled muscles made correct predictions of the forces produced by individual muscles and combinations of muscles. Finally, we verified our use of distributions of CEP locations. Figure 12 shows that we can correctly predict the distributions of CEPs that are observed during four commonly occurring muscle coactivations.

Having verified the predictive accuracy of our force field models, we proceeded to ask, where can each combination of muscles stabilize the limb and how likely is it to do so? We approached this question by examining random activations of the muscles in each possible muscle combination. In this way, we could estimate the probability of producing a stabile posture (a CEP), and the locus of limb postures at which those CEPs occurred. Our analysis has led to the surprising result that in the large space of simulated muscle combinations there exists a well-defined subset of synergies which will stabilize the limb despite activation noise, muscle fatigue, and other uncertainties-and these synergies stabilize the limb at predictable, restricted locations in the workspace. So, according to our model, these robust muscle synergies can be depended on to get the limb to the right place. We observed that the robust muscle synergies bring the limb to four discrete locations in the workspace which are near to the four discrete CEP locations we have found to be evoked by microstimulation of the interneuronal areas of the frog's spinal cord (Bizzi, Mussa-Ivaldi, \& Giszter, 1991; Giszter, MussaIvaldi, \& Bizzi, 1993; Loeb, Giszter, Borghesani, \& Bizzi, 1993; Saltiel, Tresch, \& Bizzi, 1998). We conclude that the frog, at least, may make use of noise-resistant muscle synergies to correct for the vagaries of the force output of individual muscles.

\section{RESULTS}

Model force fields of 16 hindlimb muscles are shown in Figures 1, 2, 3, 4, 5, and 6. We have grouped the muscles into six types according to the similarities that we see in these models. ${ }^{2}$ Figure 1 shows the Hip Extensor/Knee Flexor muscle type. These muscles (AD, RI, and ST) extend the hip and flex the knee. Figure 2 shows the Body Flexor muscle type. These muscles (BI and SA) predominantly flex the knee. Figure 3 shows the Rostral Flexor muscle type. These muscles (IP and PT) predominantly flex the hip. Figure 4 shows the Half Flexor muscle type. These muscles (ADl, GA, and $\mathrm{RA}$ ) flex the hip and/or the knee, and produce no force when the hip is flexed. Figure 5 shows the Hip Extensor muscle type. These muscles (QF and SM) predominantly extend the hip. Figure 6 shows three muscles acting as lateral extensors. These muscles ( $\mathrm{PE}, \mathrm{VE}$, and $\mathrm{VI}$ ) extend the knee and may flex the hip as well. ${ }^{3}$

Inspection of Figures 1, 2, 3, 4, 5, and 6 indicates that no muscle has an equilibrium point in the limb's workspace. Neither the individual muscles' recorded force fields, nor the models appeared to be stable in the workspace. Some muscles display near-zero forces in the part of the workspace towards which their forces point. In these muscles, the general flow of the muscle's force fields appears to pull the ankle to a part of the workspace where the magnitude of the muscle's force becomes negligible. All of the half flexors (ADl, GA, and RA, Figure 4) have this property, as do RI and RIm (Figure 1), SA (Figure 2), and VI (Figure 6). Such muscles could be used by the spinal circuitry to selectively modulate forces in some parts of the workspace, without affecting the forces generated in other parts of the workspace. 


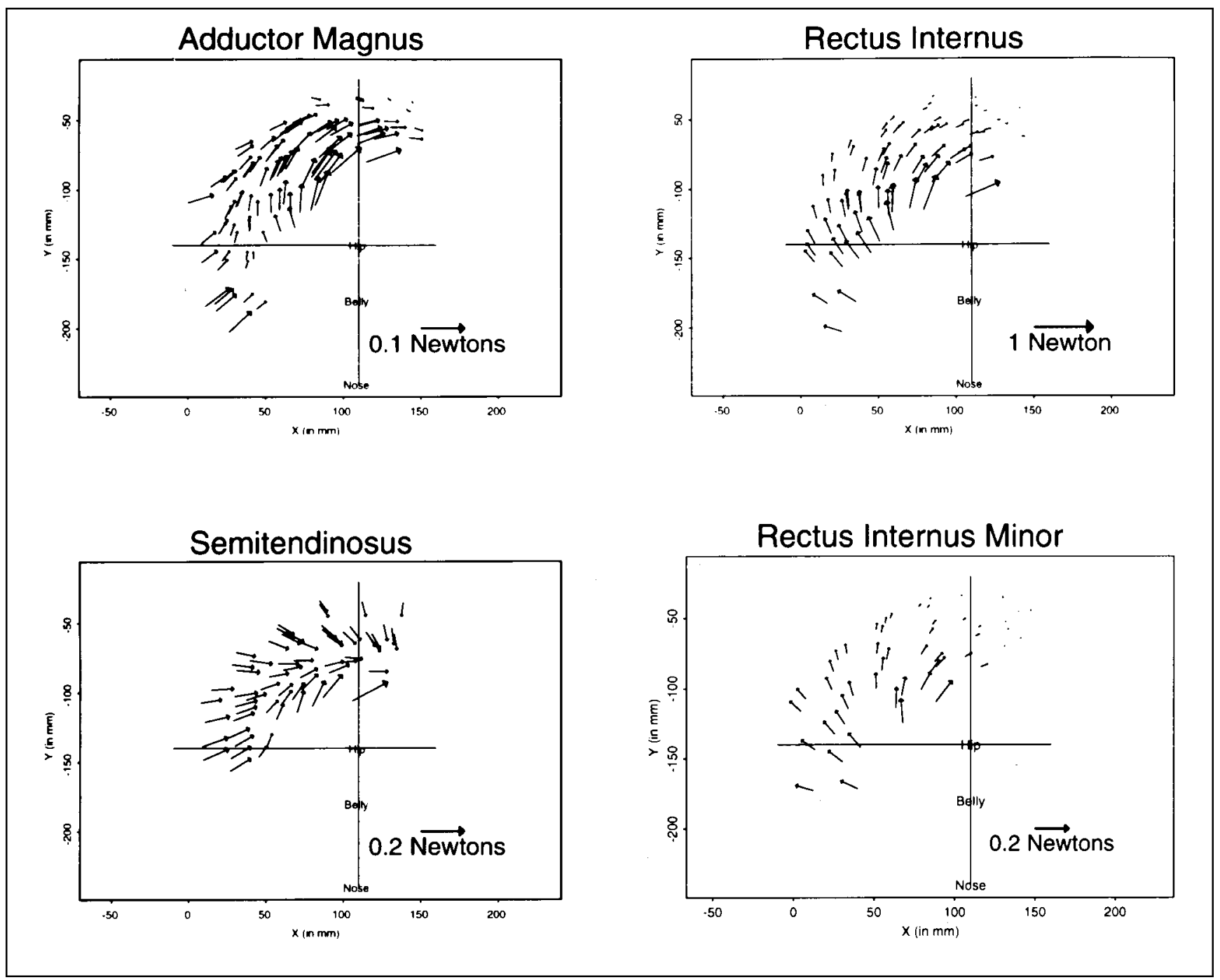

Figure 1. The hip extensor/knee flexor muscles: Adductor Magnus (AD), Rectus Internus (RI), Semitendinosus (ST), and Rectus Internus Minor (RIm) all function as hip extensors and knee flexors. Note muscles RI and RIm have zero-force regions toward which their forces point. The mean force field correlations of the models with their data force fields are 0.76 (AD, 7 frogs), 0.85 (RI, 6 frogs), 0.87 (ST, 5 frogs), and 0.91 (RIm, 3 frogs). This figure and Figures 2, 3, 4, 5, and 6 are constructed as per Figure 1B, using the modeled data at each limb position for which we collected data from any frog used in the model.

For each of the $16 \mathrm{leg}$ muscles of six frogs, we computed the correlation values between the recorded muscle force fields and the corresponding force field model for that muscle. The histogram of correlation values for 96 recorded muscles has a median correlation value of .871 (Figure 7). This histogram indicates that the model force fields have captured the main features of the experimentally derived force fields.

\section{Prediction of End-Point Force from Models and EMGs}

To validate the linear combination of muscle models, we used measured electromyographic (EMG) signals and their corresponding model muscle force fields to predict the total force observed at the limb's end-point. The EMG signals and the end-point forces were recorded during spinal microstimulation (Bizzi et al., 1991; Giszter et al., 1993). We used the EMG signals to scale the muscle model forces and summed the scaled models to generate predictions of the forces that should be observed at the ankle during the time that the EMGs were recorded (see Methods section). Since we were, in fact, measuring the forces at the ankle at the time the EMGs were recorded, we were able to compare the predicted ankle forces to the observed ankle forces. Figure 8 shows that there is good qualitative agreement between the observed and predicted forces.

In Figure 9, we quantify the degree of correlation between actual and predicted end-point forces. We found a high correlation $\left(R^{2}=.864, F(33,160)=36.9\right.$, $p<.001)$ between predicted and actual endpoint force orientations for 244 spinal stimulation trials in three animals. There are 33 free parameters in the $F$-statistic because 11 EMG gains were computed for each of the three animals in the dataset. Ninety-five percent of the 
Biceps

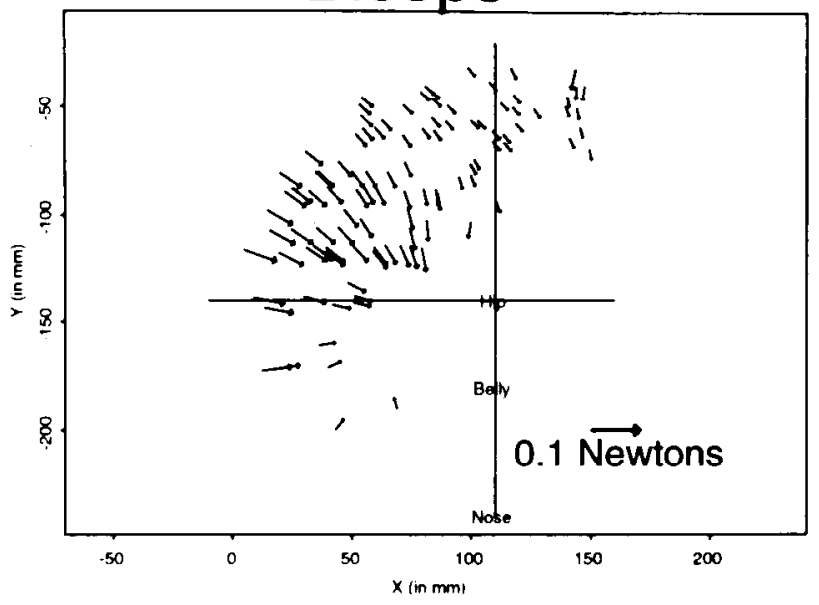

\section{Sartorius}

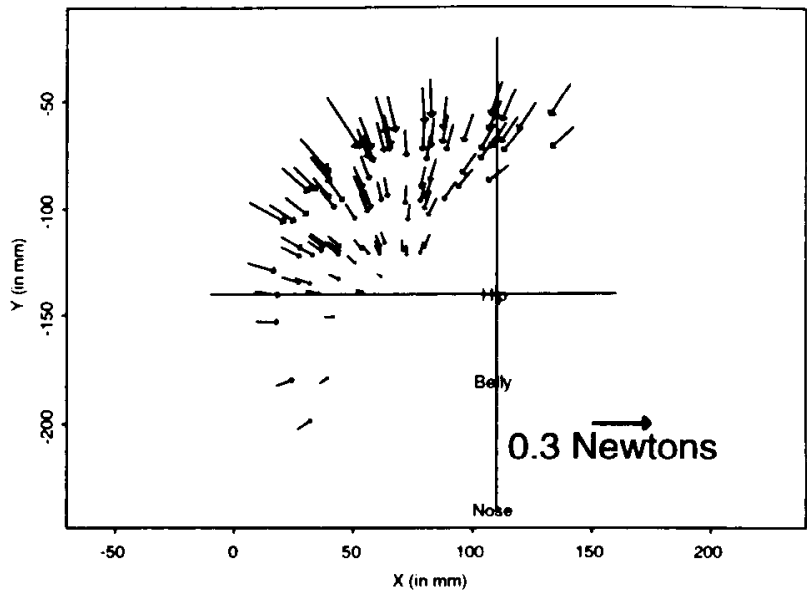

Figure 2. The body flexor muscles: Biceps (BI) and Sartorius (SA) function predominantly as knee flexors. The flow of forces are reminiscent of the flexion-to-body behavior of spinalized frogs. The mean force field correlations of the models with their data force fields are 0.81 (BI, 8 frogs) and 0.85 (SA, 9 frogs).

trials in the figure have a prediction error strictly less than $45^{\circ}$. Thus, we are able to account for a large portion (86\%) of the variance in peak force orientation using our muscle models and observed EMG signals.

\section{Simulated Synergies: Combining Muscles' Force Field Models}

The main result of this work is a new method for analyzing muscle synergies. We simulated the forcefields resulting from all possible combinations of 16 model muscles. Our simulation resulted in an estimated dis- tribution of CEPs for the $2^{16}=65,536$ combinations of muscle's force fields that are possible with 16 muscles. As activation coefficients for the muscle models, we used random numbers [0 to 100] instead of using the EMGs used in the previous section. The algorithm for the muscle combination simulation is as follows (see Methods section):

1. Select a muscle combination, $\mathrm{C}$

2. Estimate CEP distribution of $\mathrm{C}$ (Figure 10):

3. Weight each muscle in $\mathrm{C}$ by a random value.

4. Sum the weighted muscle model force fields.

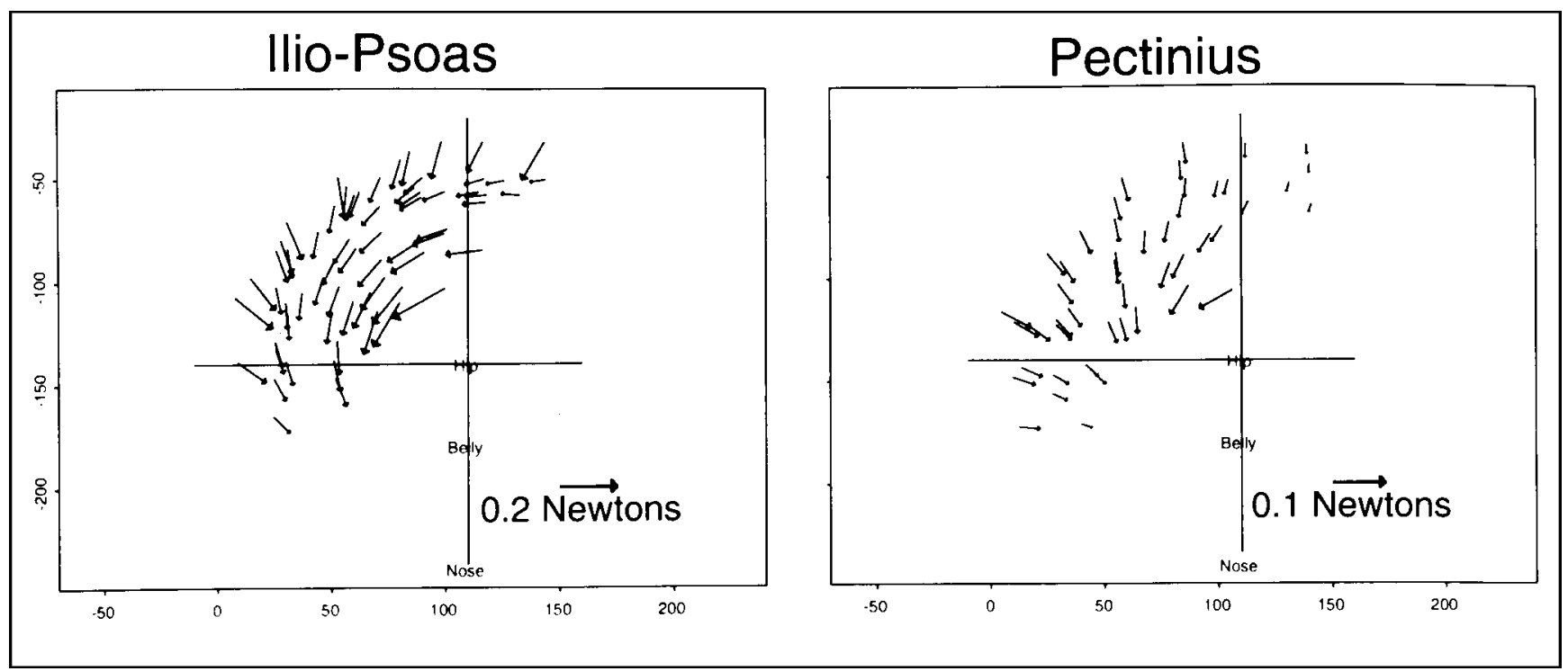

Figure 3. The rostral flexor muscles: Ilio-Psoas (IP) and Pectineus (PT) function as hip flexors. The flow of forces are reminiscent of the preparatory, rostral flexion phase of the back wiping behavior of spinalized frogs. The mean force field correlations of the models with their data force fields are 0.78 (IP, 5 frogs) and 0.90 (PT, 3 frogs). 


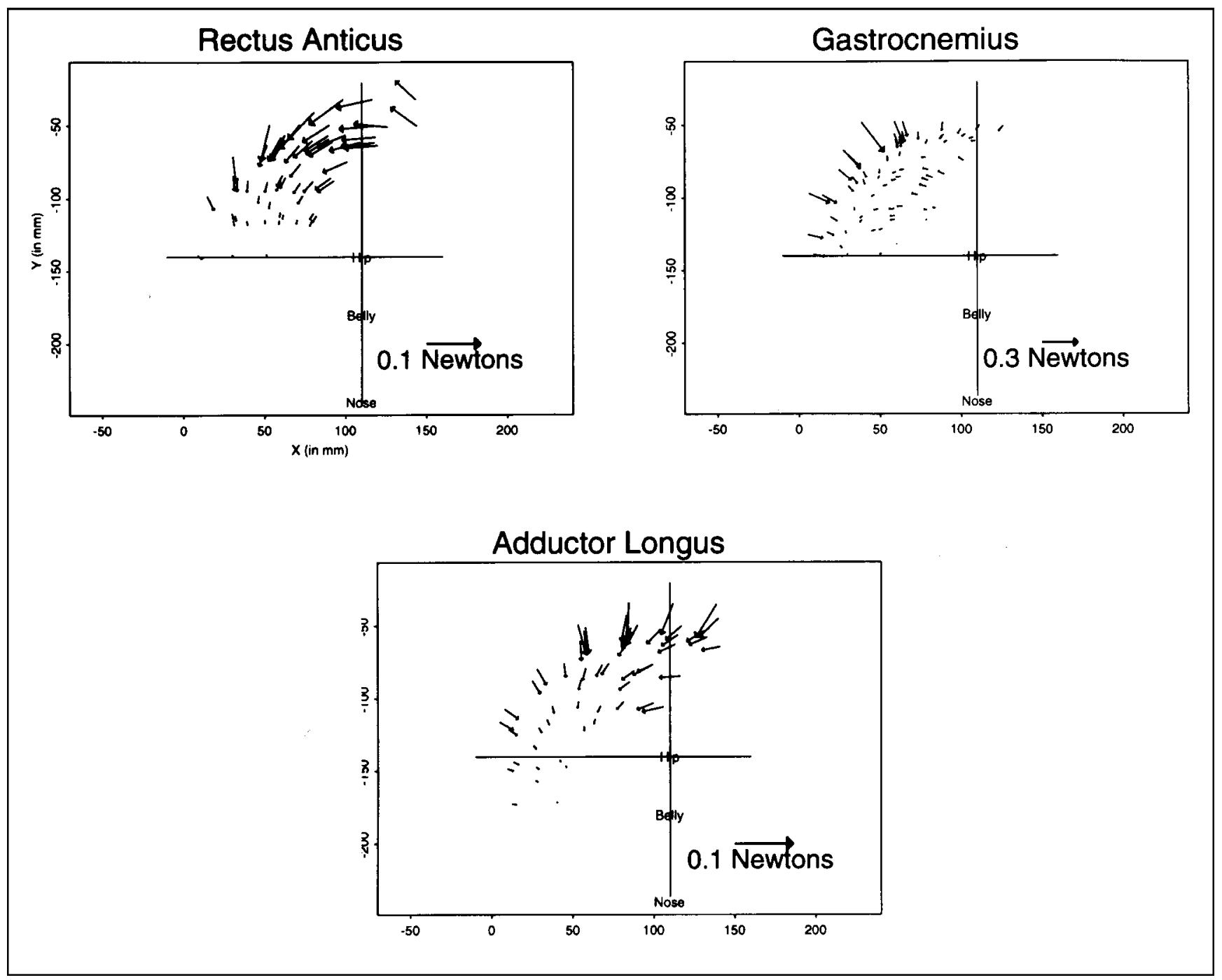

Figure 4. The half-flexor muscles: Rectus Anticus (RA), Gastrocnemius (GA), and Adductor Longus (ADl) all function as hip or knee flexors. All 3 muscles have vanishing moment ( 0 forces) when the hip is flexed. The mean force field correlations of the models with their data force fields are 0.90 (RA, 6 frogs), 0.84 (GA, 8 frogs), and 0.72 (ADl, 4 frogs).

5. Does resulting force field converge? Where?

6. Store the answers and return to step 2.

7. Compute and store the $(x, y)$ location of the joints space centroid of the CEP locations determined in steps 2 through 6 .

The loop in step 2 was typically performed $50-500$ times for each muscle combination. Step 2 results in an estimate of the probability of convergence and a distribution of the convergence locations for the given muscle combination, C (See Figure 10D).

In Figure 11, we plot the stored CEP centroids produced by the muscle combination simulation for thousands of different muscle combinations. Although the CEP locations for a single muscle combination are often scattered throughout the workspace, we have summarized each combination's CEP distribution with a single dot (at the centroid of its CEP distribution) for the purpose of this figure.
In Figure 11A, we show the centroids of reliable muscle combinations. These are muscle combinations that produced a convergent force field with $95 \%$ or more of the random activation patterns we tried. There are 14,256 reliable combinations out of 65,536 possible combinations. So, there are 14,256 dots in Figure 11A. Each dot is the centroid of one muscle combination's distribution of convergent equilibria.

In Figure 11B, we show the centroids of the localized muscle combinations. These are the combinations that produced CEPs that always fell within some 5000 square degree area in the workspace (approximately $70^{\circ}$ maximum excursion in hip angle and knee angle). The cutoff, 5000 square degrees, is one standard deviation below the mean localization value in Figure 15B.

In Figure 11C, we have plotted the 909 (out of 65,536) centroid locations of combinations which we have named robust. These combinations are "reliable" and "localized": They produce CEPs for at least 95\% of the 


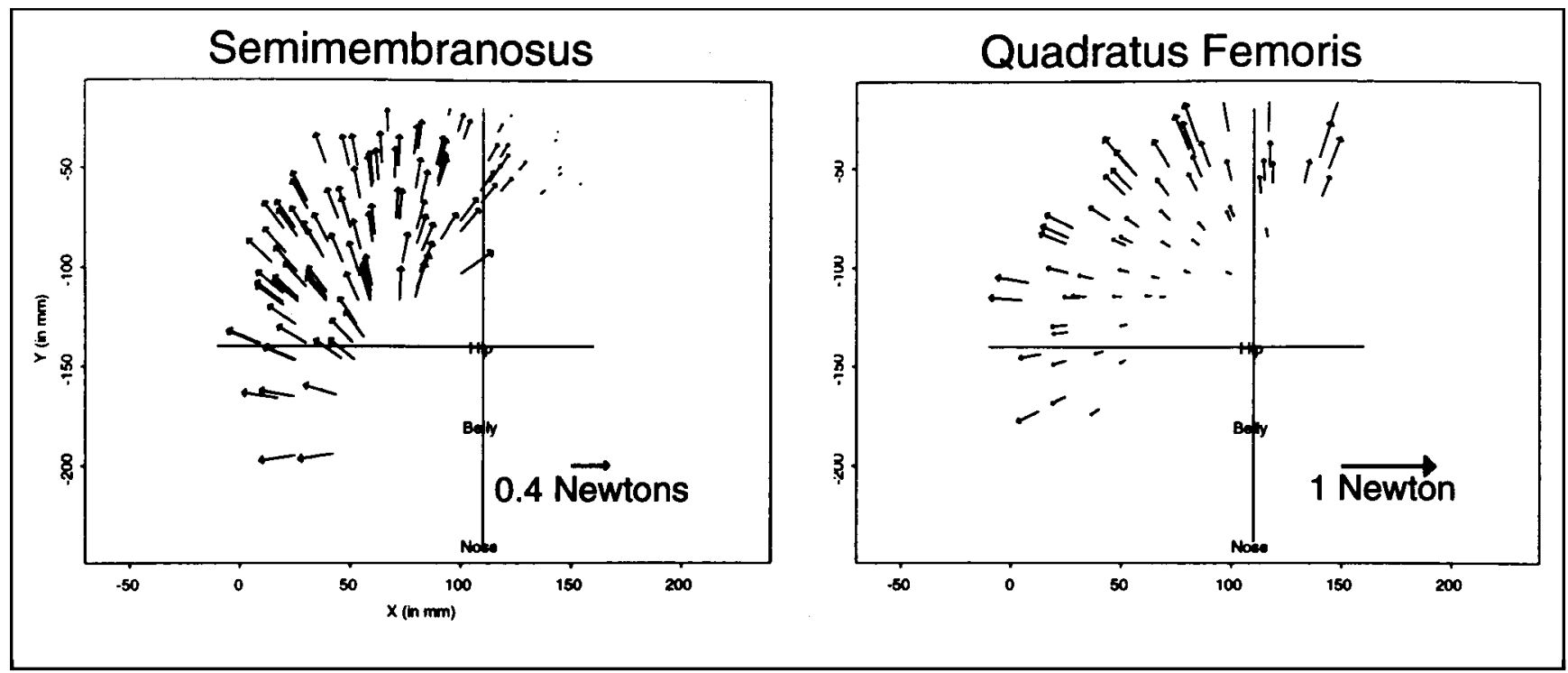

Figure 5. The hip extensor muscles: Semimembranosus (SM) and Quadratus Femoris (QF) function as hip extensors. The mean force field correlations of the models with their data force fields are 0.89 (SM, 8 frogs) and 0.83 (QF, 4 frogs).
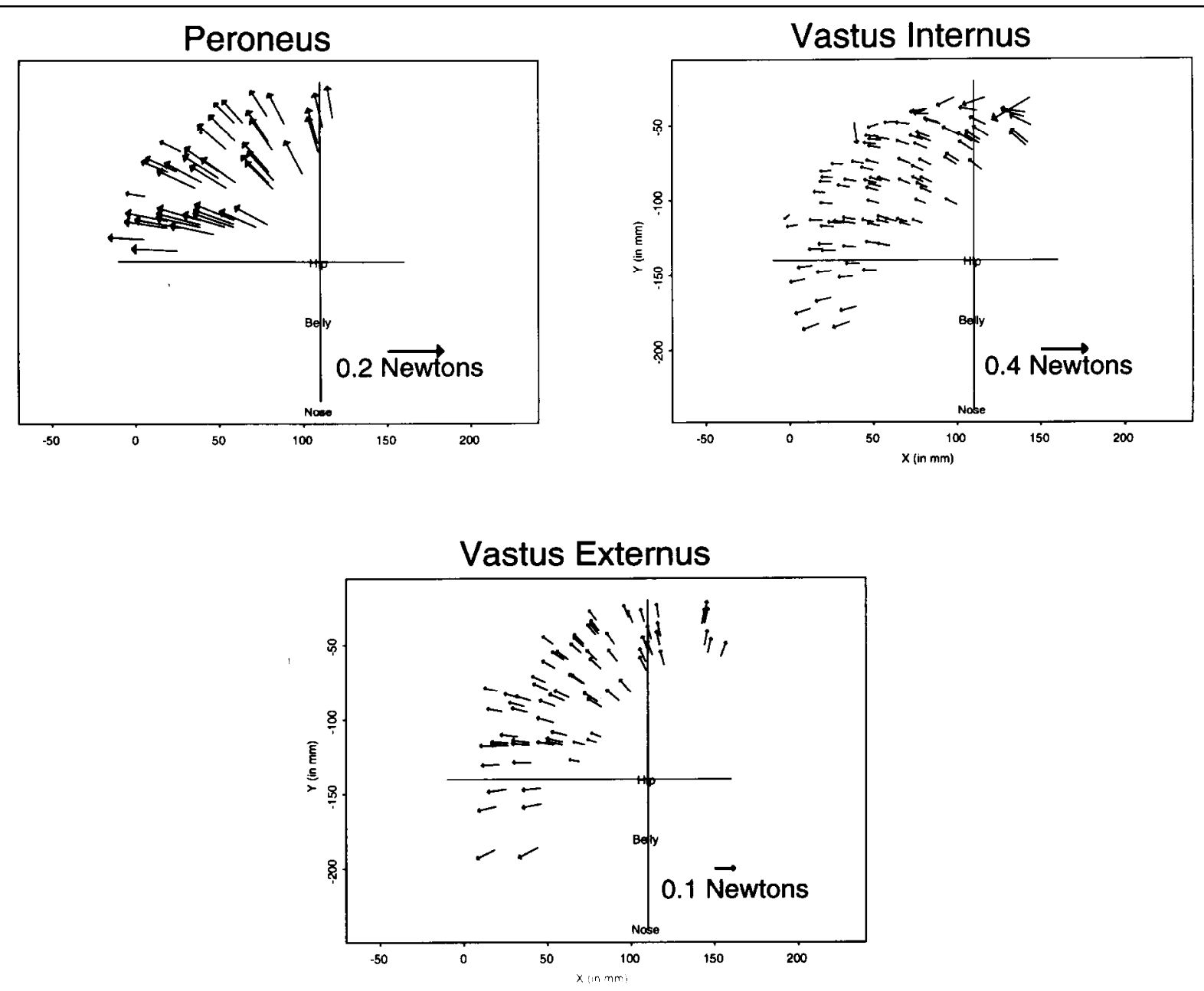

Figure 6. The lateral extension muscles: Peroneus (PE), Vastus Internus (VI), and Vastus Externus (VE) all function as knee extensors with muscles VE and VI also acting as hip flexors. The mean force field correlations of the models with their data force fields are 0.78 (PE, 5 frogs), 0.62 (VI, 8 frogs), and 0.90 (VE, 6 frogs). 


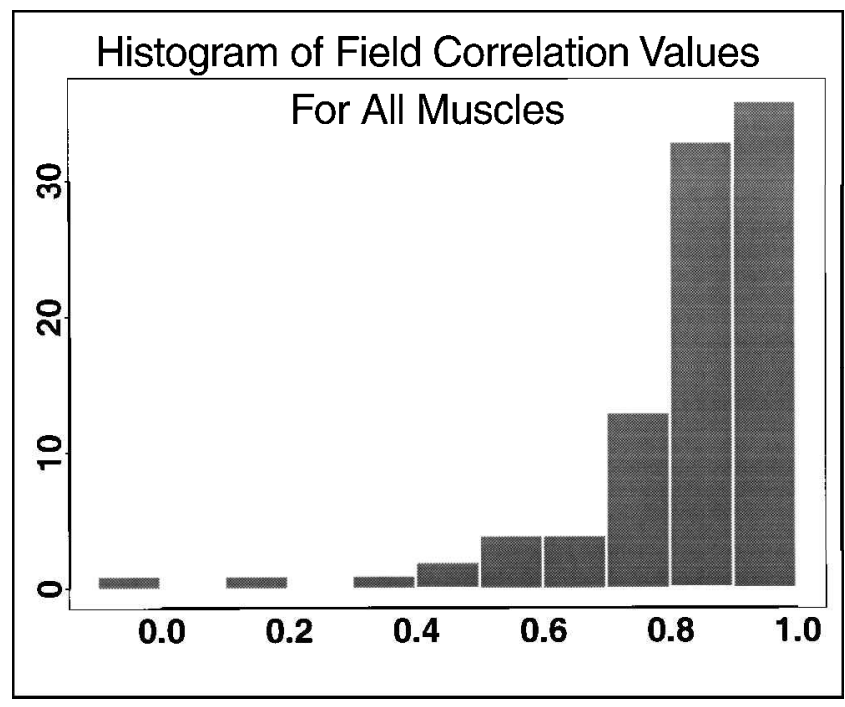

Figure 7. Correlation between recorded muscle force fields and muscle models: Histogram showing force field correlation value on the $x$ axis and the number of force fields on the $y$ axis. This histogram provides an overview of the mean correlation values given in the legends of Figures 1, 2, 3, 4, 5 and 6. For each frog and each muscle we computed the correlation between the measured force field for that muscle and the model of that muscle constructed from the measurement of all the frogs. The figure shows that 35 of the 96 recorded muscle force fields correlated with their model force fields at a level of $90 \%$ or greater.

random activation patterns we tried, and the resulting CEPs were entirely contained within some 5000 square degree portion of the workspace.

The robust combinations displayed in Figure 11C are not uniformly distributed, but cluster in three or, possibly four, groups. It is of interest that there is a degree of correspondence between CEPs of these robust combinations and the CEPs of force fields evoked by microstimulation of the spinal cord (Bizzi et al., 1991; Giszter et al., 1993). The degree of correspondence is even more marked when more restrictive criteria of robustness are applied. In Figure 11D, the criterion of reliability was increased to $98 \%$, and the localization cutoff was decreased to 1600 square degrees ( $40^{\circ}$ per joint).

\section{Comparison of Simulated and Experimental Force Fields}

The rough correspondence between the CEPs of the robust combinations and the CEPs of spinally-evoked force fields raises the question whether the muscles that implement the spinally-evoked fields belong to the class of robust synergies. To address this question, we applied the simulation procedure to the groups of muscles that were experimentally found to be active during spinal microstimulation.

In Figure 12, we see the degree of overlap between actual and simulated CEP distributions for the empirical muscle synergies. For example, in Figure 12 (upper right) the simulated distribution for the muscle combi- nation ( $\mathrm{AD}, \mathrm{GA}, \mathrm{PE}$, and $\mathrm{RI})$ is represented by one red dot for each simulated CEP. These red dots completely cover the observed distribution of CEP locations evoked by microstimulation trials producing Caudal Extension (area E, see Saltiel et al. 1998) force fields. We have observed that Caudal Extension force fields are associated with the muscle combination (AD, GA, PE, and $\mathrm{RI}$ ), and random activation of these five muscle models produced the scatter of CEPs shown in red in Figure 12 (upper right). The mean of the random, computed CEPs substantially overlaps the observed CEP distribution, so the predictive accuracy of the muscle models is again confirmed. However, the distribution of the computed CEPs is too broad for this combination to be robust by the definition of Figure 11C.

Similarly, in Figure 12 upper left, lower left, and lower right, the computed CEP distribution overlaps the observed CEP distribution for the set of muscles tested. Thus, we were able to confirm (qualitatively) the predictive accuracy of our estimated CEP distributions.

A detailed inspection of Figure 12 reveals that the simulation of experimentally-evoked synergies leads to a high density of their equilibria in certain regions of the workspace. For example, in Figure 12 (upper right), we found that the broadness of the scatter of equilibrium points is mostly attributable to the muscle SA. When SA is weakly activated, the scatter of CEPs is small, because the other muscles (that is, all muscles in the combination except for SA) are a robust muscle synergy. Conversely, when the activation of muscle SA is large relative to the other muscles in the combination, the CEPs are more broadly scattered. This logic applies to all of the panels in Figure 12. They all show dominant clustering at the locus of a robust synergy with weak additional scatter due to the other muscles. In contrast to the simulations, the experimental CEP distributions are less broadly scattered, and two are restricted to the regions of dense clustering (Figure 12, upper left and Figure 12, upper right). The range of muscle activations induced by the stimulation of spinal interneurons may be particularly constrained for those muscles that tend to induce a large excursion of the CEP.

\section{DISCUSSION}

This paper has four key points:

1. We present a novel method for exploring the action of muscle synergies. We characterize muscle synergies as probability distributions of (in particular) CEP locations in the limb's workspace.

2. We have used CEP distributions as a tool to examine a few naturally occurring synergies. In particular, we asked if the spinal cord uses muscle synergies that are robust to activation noise.

3. We have collected new data on the action in the $x, y$ plane of 16 hindlimb muscles of the frog. 
Figure 8. Forces recorded at the ankle produced by spinal stimulation are compared with model forces: (A) The traces along the top row are (from left to right) the observed $(x, y)$ forces, the predicted $(x, y)$ forces, and the filtered EMG signal from the following muscles: RA, RI, AD, SM, ST, VI, BI, SA, VE, TA, and GA. (B) Three more observed and predicted force traces from the same animal.
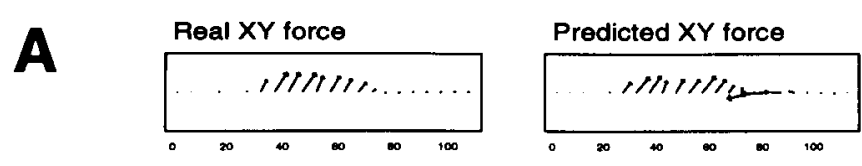

RI

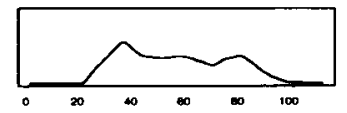

AD

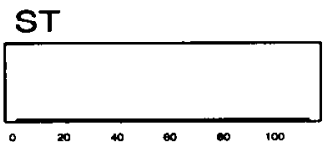

SA

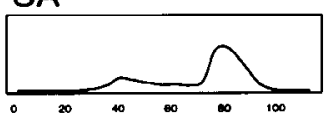

GA
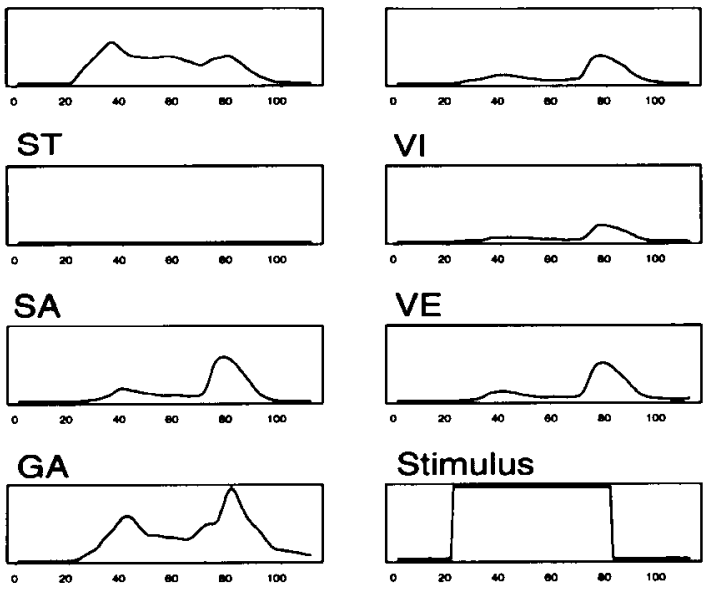

VI

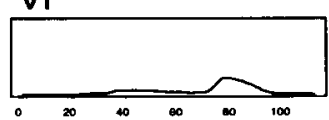

VE

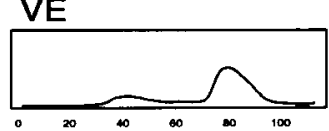

Stimulus

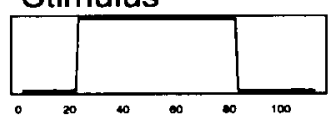

Real XY force

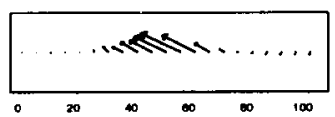

Predicted XY force

B
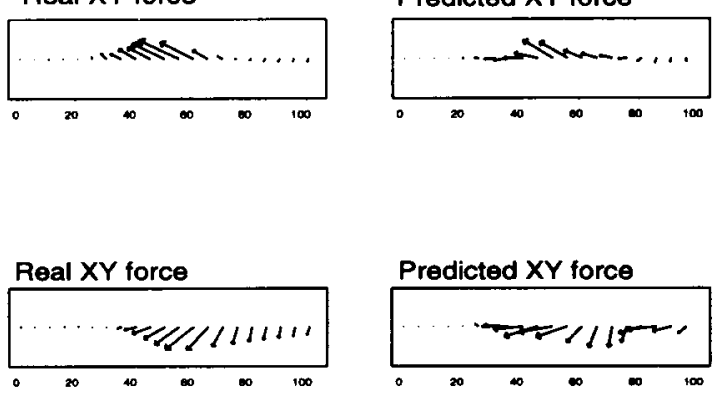

Real $X Y$ force
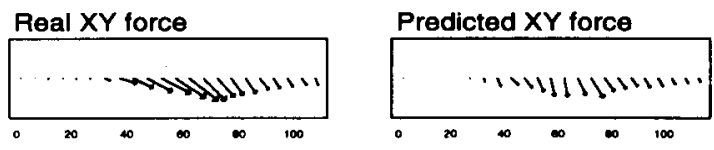

RA

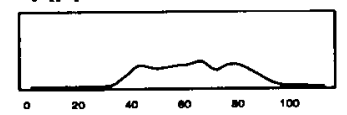

SM

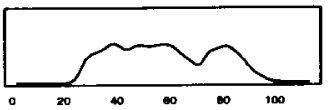

BI

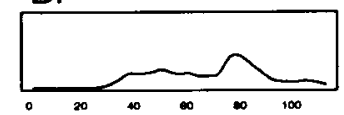

TA

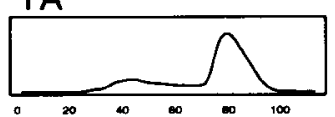

None of the muscles could individually stabilize the limb.

4. We developed a new method for modelling the force produced by leg muscles.

\section{The Distributions Associated with Muscle Synergies}

We used combinations of muscle models to associate each possible muscle synergy with a probability distribution of CEP locations in the workspace. By converting synergies to distributions, we were able to examine the entire set of muscle synergies quantitatively, because we were able to compare the distributions. For example, once we determined the likelihood of convergence for each muscle combination, we were able to select the synergies that had a high likelihood of convergence relative to all the other synergies. This general method can be extended to many other features of interest: Here we have focused on distributions of equilibrium points, but it is also possible to examine distributions of forces, dynamic variables, and so forth.

\section{Does the Spinal Cord Use Robust Synergies?}

Electrical or chemical microstimulation of the premotorneuronal circuitry of the spinal cord of the frog produces precisely balanced contractions in 


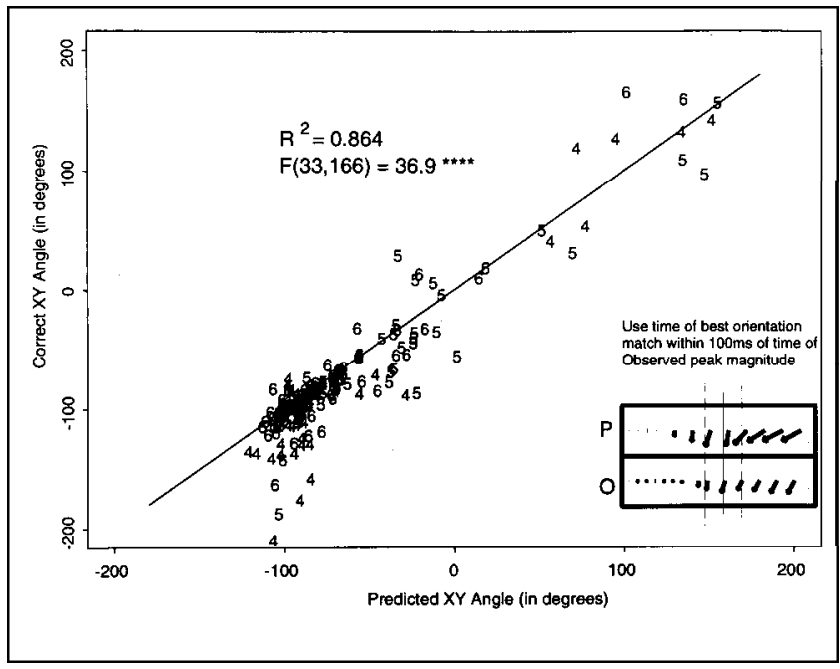

Figure 9. Summary statistics for force predictions from a large database: We used 244 spinal stimulation trials from three animals to test the accuracy of force predictions from EMG signals. Fifteen percent of the trials from each animal were used to create 11 EMG gain factors per animal (one gain for each recorded muscle in each animal). These trials were then removed from the dataset. Thus, there were no parameters determined by the data in this figure. The remaining 200 trials are shown here. The trials are labeled " 4 ", " 5 ", and "6" after their animal labels. The inset illustrates the method used to compare Predicted and Observed force traces (see Methods).

groups of leg muscles. These synergistic contractions generate forces that direct the limb's end-point toward an equilibrium point in the workspace of the leg.

To record the spatial variations of forces that are generated by the leg muscles, Bizzi et al. (1991), Giszter et al. (1993), and Saltiel et al. (1998) stimulated sites in the spinal cord and recorded the direction and amplitude of the elicited isometric force at the ankle. They found that the elicited force vectors varied as they placed the limb at different locations. The collection of the measured forces corresponded to a force field.

Different groups of leg muscles were activated as the stimulating electrodes (or pipettes) were moved to different loci in the lumbar spinal cord in the rostrocaudal and mediolateral direction. After mapping most of the premotor area of the lumbar cord, Bizzi et al. (1991), Giszter et al. (1993), and Saltiel et al. (1998) reached the conclusion that there were at least four areas from which distinct types of convergent force fields could be elicited.

In this paper, we performed an exhaustive simulation of the limb stabilizing abilities of all combinations of 16 muscles. Within these 65,536 possible muscle synergies, we found a small subset that should, according to our model, be robust in the face of the various sources of noise with which the motor control system must contend. In particular, these robust synergies are "reliable" and "localized". They are reli- able, because they produce convergent force fields for almost all of the random patterns of activation we tried. Thus, we expect these synergies to stabilize the limb even if (for example) one of the muscles is fatigued. The robust synergies are localized, because their CEP locations fall within a relatively small area in the workspace. Thus, we expect these synergies to stabilize the limb at a predictable location even if (for example) changes in ambient temperature cause the surface muscles to be less effective than the deeper muscles. Reliability and localization combined make the robust synergies apparently useful as building blocks of limb stability.

The composition and the behavior of the robust synergies is correlated to the composition and behavior of the synergies that are evoked by electrical and chemical stimulation of the spinal cord. Compositionally, the evoked synergies always contain at least one robust synergy. Behaviorally, the evoked synergies produce CEP locations in the same portions of the leg's workspace as the robust synergies. It remains to be investigated whether the robust synergies are used as building blocks, as these findings suggest. The preservation of stability in the face of variable effects of control signals (due to muscle fatigue, muscle temperature, limb velocity, muscle stiffness, and immediate activation history) presumably plays an important and fundamental role in motor control systems.

\section{Models of the Leg's Muscles}

We described a statistical model of the frog's muscles which accurately predicts the orientation of the force vectors generated by the activation of individual leg muscles. The models utilized in this study were constructed by least-squared-error parameter estimation techniques. This approach has made it possible to avoid difficult measurement problems. Previous muscle models were based on actual measurements of muscle length and muscle's moment arm derived from cadavers (Loeb, He, \& Levine, 1989; Wickland, Baker, \& Peterson, 1991). These measurements rely heavily on mathematical simplifications of the intricacies of muscle geometry in order to compute muscle length as a function of the joint angles for each modeled muscle (Buchanan, Almdale, Lewis, \& Rymer, 1986; Wickland et al., 1991; Winters \& Stark, 1988; Zajac and Winters, 1990). However, it is unclear how well muscle geometric parameters generalize across subjects, and these invasive measurements are not practical in all settings. In this study, we have instead constructed muscle length functions from measurements of endpoint forces induced by muscle stimulation at different joint angles (see Methods Section).

We utilized our muscle models to predict the forces recorded at the ankle following spinal microstimula- 


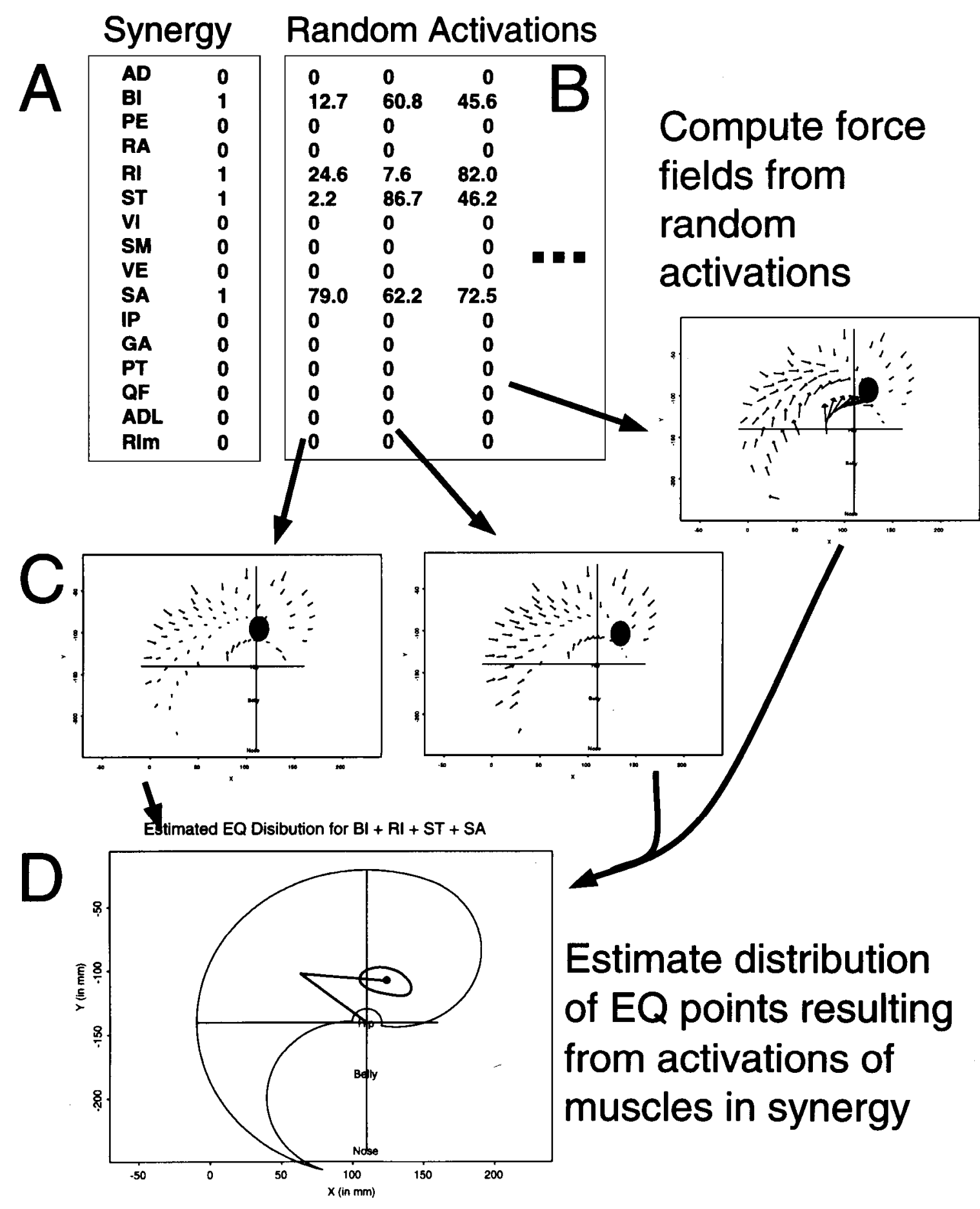

Figure 10. Monte Carlo simulation analysis of a single muscle combination: The force fields produced by random activations of muscles are computed so as to summarize each muscle combination's distribution of CEPs. (A) The binary vector encoding BI+RI+ST+SA. (B) Three random activation patterns conforming to the $\mathrm{BI}+\mathrm{RI}+\mathrm{ST}+\mathrm{SA}$ muscle combination. All muscle activation values are zero, except for the four muscles in the combination. The non-zero activation values are random numbers chosen from a uniform distribution over [0, 100]. (C) The three force fields resulting from the three activation patterns in (B). The force fields are computed in the manner of Figure 7 , using the random activation values instead of gains and EMGs. For example, the forces in the first (left-most) force field are 12.7 times the forces of the BI muscle model, plus 24.6 times the forces of the RI muscle model, and so on. The three illustrated force fields all converged; their equilibria are indicated by the large dots. (D) Distribution of the CEP locations for BI+RI+ST+SA. We sampled 2000 activation patterns for $\mathrm{BI}+\mathrm{RI}+\mathrm{ST}+\mathrm{SA}$, and $66 \%$ (1322) of those sampled activation patterns led to convergent force fields. A stick-figure leg is shown with its ankle at the centroid of the distribution of the CEP locations. The ellipse surrounding the centroid has major and minor axes given by twice the (joint space) standard deviation. The whale-like shape surrounding the stick-figure leg is the boundary of the workspace (hip $>-110^{\circ}$ and $<80^{\circ}$; knee $>10^{\circ}$ and $<170^{\circ}$ ). 


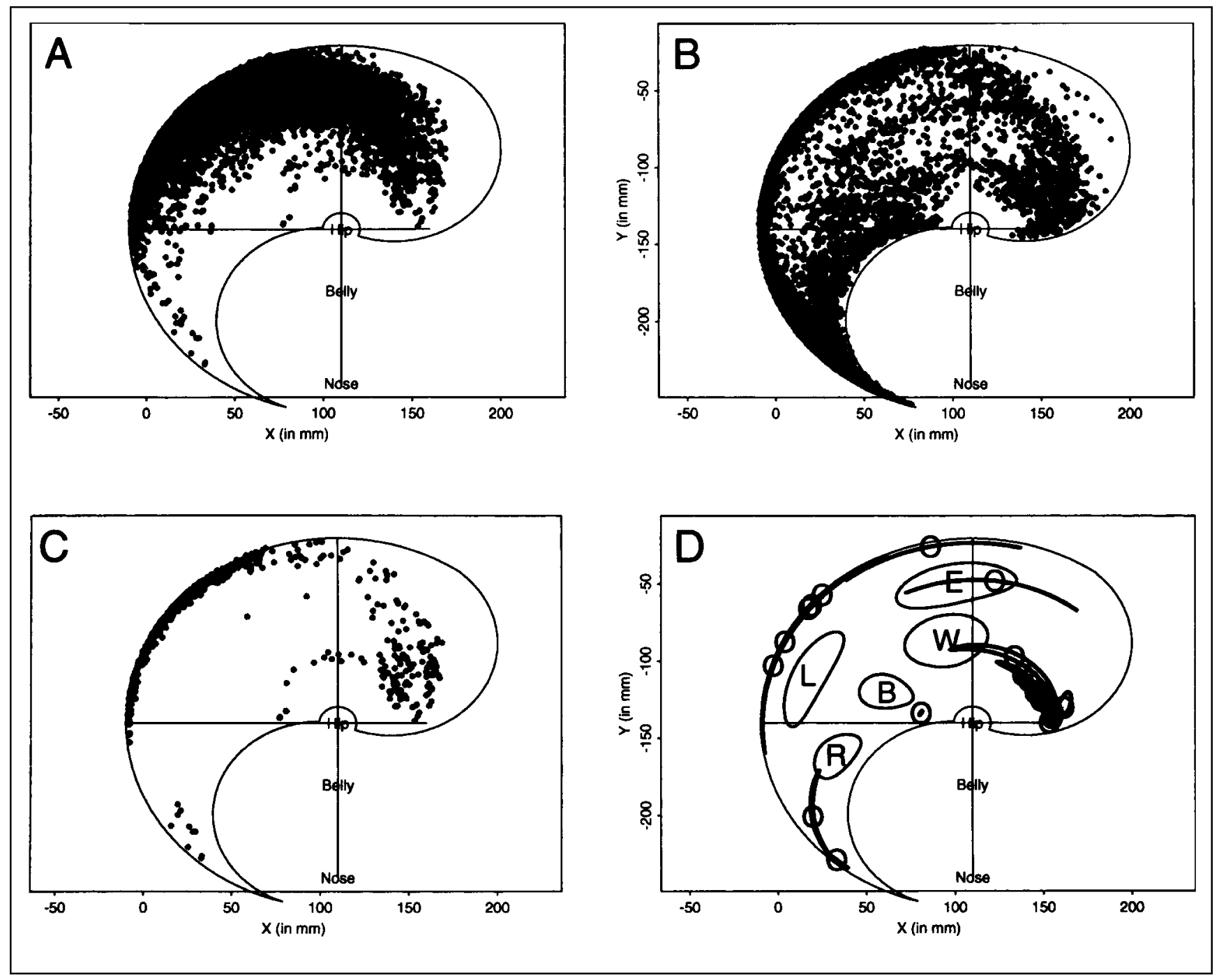

Figure 11. Reliable, localized, and robust CEP distributions: The centroids of CEP distributions are shown. (A) The 14,256 centroid locations of the reliable muscle combinations. These centroids lie away from the body, because the extensor muscles are stronger. (B) The 9538 centroid locations of the localized muscle combinations. (C) The 909 centroid locations of the robust muscle combinations. Points are plotted here only if they are plotted in both (A) and (B). (D) The 23 combinations fitting a (reliability, localization) cutoff of (98\%, 1600 square degrees). Each of these 23 robust distribution is drawn here with an "O" at its centroid within an ellipse covering \pm 1 SD in CEP location. The CEP distribution ellipses observed during spinal microstimulation are also shown for comparison. These ellipses are labelled R for Rostral Flexion; B for Body Flexion; W for Wipe; E for Caudal Extension; and L for Lateral Extension.

tion. Previous investigations (Mussa-Ivaldi, Giszter, \& Bizzi, 1994) showed that the simultaneous activation of two muscles lead to a force field which is the vector sum of each muscle's force field. In this investigation, we needed to consider the concurrent mechanical effect of several muscles. In order to asses the validity of the vector summation with such muscle populations, we have used the EMG signal measured in each muscle. We simulated the net spinal field as the sum of muscle fields, each one scaled according to the amplitude of the EMG measured in the actual spinal stimulation. Our results indicate that there is indeed an excellent agreement between measured and predicted forces. This finding demonstrates that it is possible to model the activation of multiple muscles as a weighted combination of the empirically derived muscle models.

\section{Conclusion}

We have developed a method that allowed us to identify muscle synergies with special properties out of a large set of possible muscle combinations. This method, which is based on the modelling of the force generated by individual muscles, will allow us to examine the robustness of muscle combinations not only during posture (as we did here), but also in a variety of other circumstances, such as reflex activation, and centrally generated movements. Finding properties of robustness of synergies in these motor behaviors will open the way 


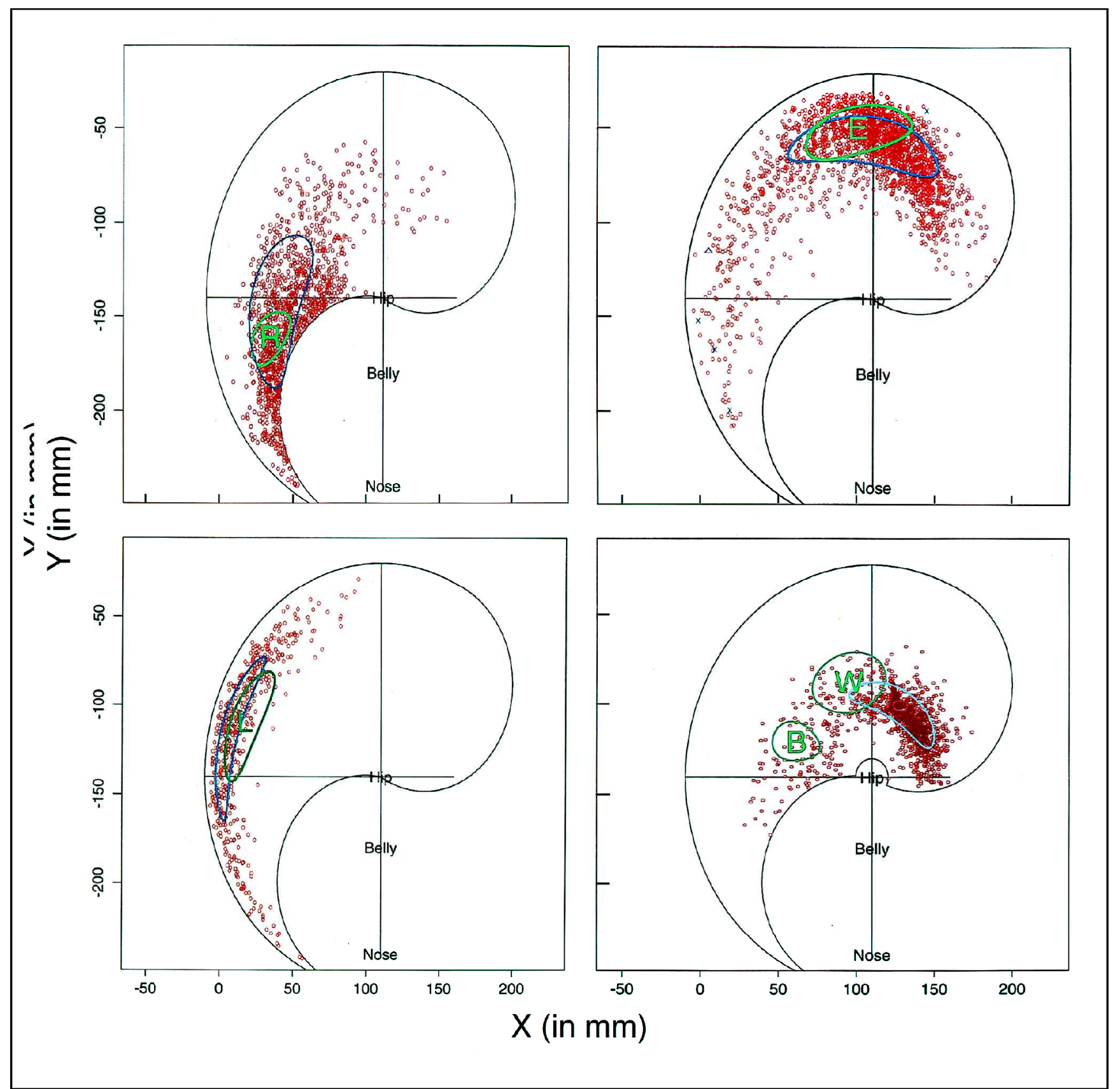

Figure 12. (Upper Left) The rostral flexion combination: There are seven muscles in this combination: BI, GA, IP, PE, RA, SA, and ST. We have observed Rostral Flexion hindlimb forces in conjunction with this muscle combination. The distribution of red, computed CEPs (summarized in light blue) overlaps the green distribution of observed Rostral Flexion equilibrium points. The red distribution has reliability $29 \%$ and localization 21,837 square degrees. The sub-combination BI+GA+PE+RA+SA is robust. (Upper Right) The caudal extension combination: There are five muscles in this combination: AD, GA, PE, RI, and SA. We have observed Caudal Extension hindlimb forces in conjunction with this muscle combination. The distribution of red, computed CEPs covers the green distribution of observed Caudal Extension CEPs. The red distribution has reliability $99.3 \%$ and localization 25,440 square degrees. The sub-combination AD+PE $+\mathrm{RI}$ is robust. (Lower Left) The lateral extension combination: There are 10 muscles in this combination: GA, IP, PE, RA, SA, and VI are strong; AD, RI, SM, and VE are weak. We have observed Lateral Extension hindlimb forces in conjunction with this muscle combination. The distribution of red, computed CEPs overlaps the green distribution of observed Lateral Extension equilibrium points. The red distribution has reliability $99.4 \%$ and localization 15,594 square degrees. This large combination of muscles has several robust sub-combinations. (Lower Right) The hindlimb wipe combination: There are seven primary muscles in this combination: AD, BI, IP, RA, RI, SA, and ST. We have observed both Body Flexion and Hindlimb Wipe forces in conjunction with this muscle combination. The distribution of red, computed CEPs overlaps the green distributions of observed Body Flexion and Hindlimb Wipe equilibrium points. The red distribution has reliability $82 \%$ and localization 15,904 square degrees. The sub-combination $\mathrm{AD}+\mathrm{RA}+\mathrm{RI}+\mathrm{SA}+\mathrm{ST}$ is robust. 
to the study of dynamic stability, which is an important and distinct property of the vertebrate motor control system.

\section{METHODS}

\section{Surgery and Data Collection}

Twenty-four healthy adult bullfrogs (Rana Catesbiana) were anesthetized with $0.5-1.5 \mathrm{~cm}^{3}$ tricaine and spinalized anterior to the first vertebra. The skin of the right hindlimb was removed and the muscles were coated with Vaseline to keep them moist. The superficial fascia were cut to allow access to deep muscles and nerves. All large nerves were cut and removed to sever the reflex arcs and to prevent the stimulus applied to one muscle from spreading along the nerves to other muscles.

A pair of "fishhook electrodes" (stainless steel wire threaded through a syringe needle, with exposed wire at the tip bent back to form a hook (Crago, Peckham, \& Thrope, 1980)) were placed in each muscle under a dissecting microscope. Stimulus voltage was set at the start of each experiment to evoke a maximal visible muscle contraction without causing contractions in any other muscles. The surgical isolation of the muscles made it possible to verify isolated responses by visual inspection of the muscles. Another criterion for detecting the spread of activity to other muscles was the sudden change of force orientation at the endpoint of the limb. This change was readily detected when the stimulus voltage exceeded a certain threshold. The other stimulation parameters were fixed at $0.6 \mathrm{msec}$ pulses width, $600 \mathrm{msec}$ train duration, and $40 \mathrm{~Hz}$.

\section{Recording Force Fields from Individual Muscles}

Spinalized frogs were placed on a moistened molded plastic frame and secured in place by clamps attached to the pelvis. The position of the frog's hip in the apparatus and the length of its right hindlimb segments were measured. The right ankle was attached to a force sensor immediately proximal to the frog's ankle. The force sensor was attached to a frame, which enabled us to change the ankle coordinates in the horizontal $(x, y)$

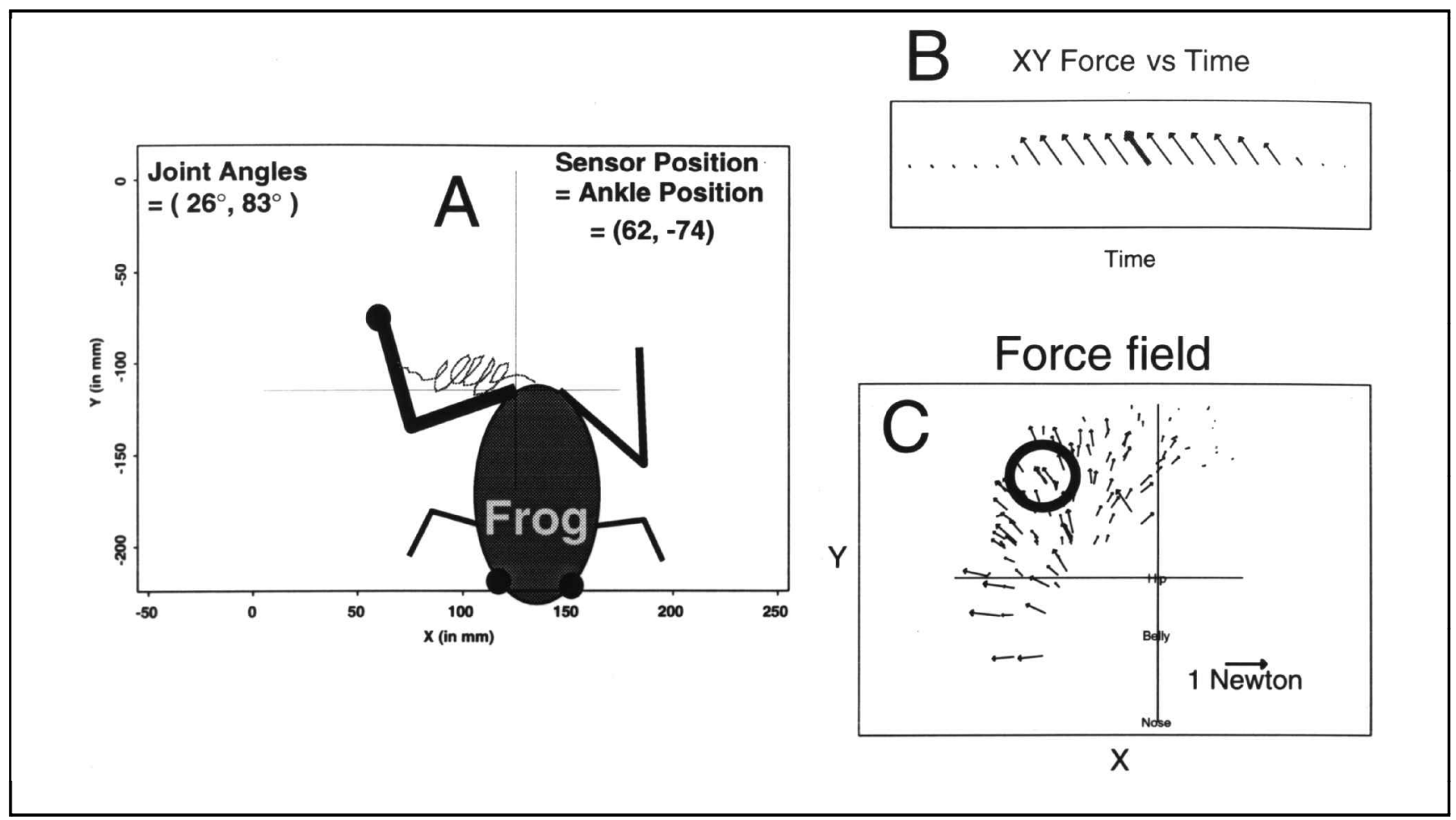

Figure 13. This figure illustrates the processing steps taken to produce a force field from the data recorded during muscle stimulation. (A) Illustration of a frog in the standard coordinate frame of our experimental apparatus. Our Cartesian coordinate system is defined by a fixed metal stand at position $(0,0)$ on which the sensor is mounted (not shown). The right hindlimb is shown at a hip angle of $26^{\circ}$, measured counter-clockwise from the horizontal, and a knee angle of $83^{\circ}$, measured counter-clockwise from full flexion. The standard frog's thigh and calf lengths are both 60 $\mathrm{mm}$. These configuration parameters correspond to an ankle/sensor position of $(62 \mathrm{~mm},-74 \mathrm{~mm})$ in the workspace. The schematic spring attached to the limb represents the muscle Semimembranosus. When the muscle is stimulated the force sensor records the translational $(x, y)$ forces. (B) Recorded $(x, y)$ force traces, induced by stimulation of a frog's Semimembranosus muscle, are plotted as a sequence of vectors. The vector with the greatest magnitude is highlighted. (C) A force field is constructed from the peak force vector at each tested limb position. The circled force corresponds to the limb position in (A) and the peak force in (B). 
plane. The $Z$ coordinate of the ankle was kept unchanged and approximately level with the hip (Figure 13A).

We measured a muscle force field by stimulating one muscle with the hind limb positioned in each of many different locations. Thus, we followed this simple procedure (Giszter et al., 1993): (1) Stimulate one muscle while measuring force. (2) Move the ankle to a new position. (3) Repeat from step 1 for the same muscle.

As the muscle contracted and relaxed in response to stimulation, the force sensor recorded $x$ - and $y$-forces as a function of time (Figure 13B). Forces were digitized and recorded in units of $0.01 \mathrm{~N}$. We initiated our force measurements 50 to $200 \mathrm{~ms}$ prior to stimulus onset. We used the first $50 \mathrm{msec}$ of the force's record to estimate the baseline force levels. We computed the active force traces by subtracting the baseline force level from the total force measured during muscle stimulation.

The active forces were time-variant, but they could be approximated as time-invariant because the active force vectors typically rose and fell along a single orientation (Figure 13B). Thus, a whole temporal sequence of active force vectors was represented as a single vector modulated by a scalar signal. We used the active force vector with the largest magnitude to represent the entire force trace. ${ }^{4}$ The peak active force vectors from each tested ankle position form a time-invariant force field, $\operatorname{FFi}(x, y)$ (Figure 13C).

To correct for the frogs' different sizes, we converted the measured forces to joint torques (Lan \& Crago, 1992). Then, we normalized the joint torques across animals by scaling each frog's torque data at a standard posture to the same value of magnitude for all frogs.
Finally, we converted the normalized torques back to forces using standardized link lengths of $60 \mathrm{~mm}$.

\section{Models Representing the Force Field of Individual Muscles}

The rationale for establishing force field models of individual muscles rests upon the need to obtain for each muscle a simple function that can be manipulated analytically. To this end, we developed statistical models using a least-squares procedure based upon the forces we recorded from individual muscles. We considered a number of different models. We tested the ability of each model type to generalize to new data using the Jackknife technique (Efron and Tibshirani, 1982). We performed the Jackknife tests on a dataset of force fields observed during the stimulation of muscles in nine frogs.

Table 1 shows the average generalization errors we found for several muscles and model types. The generalization errors were measured as root mean square torque errors and as force field correlations (Giszter et al., 1993; Mussa-Ivaldi et al., 1994). Small root mean square errors and large correlation values indicate that a model should generalize well over new data. Table 1 shows that the "virtual work" model type (described below) had the smallest errors of the five model types we compared.

\section{The Virtual Work Model Type}

Our virtual work model consists of a parameterized muscle length function and a fixed length-tension curve. We derived this model from the invariance of work

Table 1. Jackknife Errors for Five Model Types

\begin{tabular}{lcccccc}
\hline Muscle & N Frogs & Mean Torque & Linear Torque & Nine Gaussians & Gated Experts & Virtual Work \\
\hline SM & 9 & $4.32(66 \%)$ & $3.87(68 \%)$ & $4.67(77 \%)$ & $2.55(79 \%)$ & $2.27(87 \%)$ \\
SA & 9 & $4.45(73 \%)$ & $3.73(73 \%)$ & $3.69(78 \%)$ & $2.65(76 \%)$ & $2.49(79 \%)$ \\
ST & 5 & $2.22(72 \%)$ & $2.85(83 \%)$ & $2.41(87 \%)$ & - & $1.56(83 \%)$ \\
AD & 7 & $6.29(71 \%)$ & $6.2(67 \%)$ & $4.16(69 \%)$ & $1.66(66 \%)$ & $1.59(68 \%)$ \\
VI & 8 & $2.88(59 \%)$ & $3.36(52 \%)$ & $3.5(65 \%)$ & - & $2.13(55 \%)$ \\
IP & 6 & $2.85(44 \%)$ & $3.27(51 \%)$ & $2.78(58 \%)$ & - & $2.71(57 \%)$ \\
VE & 6 & $3.57(82 \%)$ & $3.63(75 \%)$ & $2.25(78 \%)$ & $2.5(78 \%)$ & $2.45(80 \%)$ \\
\hline
\end{tabular}

Root mean squared error and mean force field correlation (in parenthesis) are displayed for seven hindlimb muscles and five model types. The Jackknife technique involves fitting model parameters with data from all the frogs except one, and then using that fitted model to predict the data from the frog that was left out. This fit-and-predict operation is repeated once for each frog in the dataset in order to compute an average generalization error. The root mean squared error is the square root of the average of the square of the error. The force field correlation value of force field A and force field $\mathrm{B}$ is the correlation value for the vectors $\left(\boldsymbol{x} 1_{\mathrm{A}}, \boldsymbol{x} 2_{\mathrm{A}}, \ldots \boldsymbol{x} N_{\mathrm{A}}, \boldsymbol{y} 1_{\mathrm{A}}, \boldsymbol{y} 2_{\mathrm{A}}, \ldots \boldsymbol{y} N_{\mathrm{A}}\right)$ and $\left(\boldsymbol{x} 1_{\mathrm{B}}, \boldsymbol{x} 2_{\mathrm{B}}, \ldots \boldsymbol{x} N_{\mathrm{B}}, \boldsymbol{y} 1_{\mathrm{B}}, \boldsymbol{y} 2_{\mathrm{B}}, \ldots \boldsymbol{y} N_{\mathrm{B}}\right)$, where $\boldsymbol{x} i_{\mathrm{A}}$ is the $\boldsymbol{x}$ component of the force at position $i$ of field A. Low squared errors and high force field correlations indicate good generalization. For example, the Mean Torque model type would be expected to have a root mean square prediction error of 4.32 torque units ( $0.01 \mathrm{~N} / \mathrm{rad}$ ) on new Semimembranosus data, whereas the Virtual Work model would be expected to have an error of 2.27. $N$ Frogs is the number of animals in the dataset for each muscle. The Mean Torque model predicted joint torques as the mean of the observed joint torques. The Linear Torque model predicted torque as fitted (constant plus one linear term) function of the joint angles. The nine Gaussians model (Mussa-Ivaldi, 1992) and the Gated Experts model (Jacobs \& Jordan, 1993) are described elsewhere. They both have more free parameters than the Virtual Work model. 
Table 2. Virtual Work Muscle Model Variable Values for 16 Frog Hindlimb Muscles

\begin{tabular}{|c|c|c|c|c|c|c|c|c|}
\hline Muscle & LO & $A_{k}$ & Freq $_{k}$ & $P b i_{k}$ & $A_{b}$ & Freq $_{b}$ & $P b i_{b}$ & Scale \\
\hline$* \mathrm{AD}$ & -0.36 & 2.71 & -0.894 & 0.826 & 5.37 & 0.702 & -1.07 & 1060 \\
\hline $\mathrm{ADI}$ & -1330 & 0.353 & 1.48 & -2.5 & 1330 & 0.0234 & 0.061 & 2720 \\
\hline$* \mathrm{BI}$ & 2790 & 3340 & 0.14 & 2.14 & -160 & 2.09 & 3.65 & 3.47 \\
\hline$* \mathrm{GA}$ & 140 & 4.87 & -1.64 & 2.62 & 153 & 0.0658 & 2.73 & 454 \\
\hline *IP & 24.6 & -1.04 & 1.99 & -1.1 & 10.4 & 0.634 & 1.43 & 969 \\
\hline$* \mathrm{PE}$ & 23.9 & 7.25 & -0.888 & 2.43 & 3.86 & 0.613 & 2.55 & 1050 \\
\hline PT & -604 & 78.2 & -0.0286 & -0.0912 & 527 & 0.042 & 0.046 & 3540 \\
\hline PY & 1380 & 1570 & 0.0186 & 6.16 & 2950 & 0.0228 & 3.2 & 59.5 \\
\hline QF & -10.1 & 0.789 & -1.06 & 3.18 & -0.486 & 0.612 & 4.18 & $1.73 e+08$ \\
\hline *RA & -4940 & 0.459 & -1.1 & -2.94 & 4930 & 0.0153 & 0.03 & $4.69 e+06$ \\
\hline$* \mathrm{RI}$ & 394 & 1.13 & -1.13 & 0.832 & 403 & 0.0371 & -2.91 & 5570 \\
\hline RIm & -149 & -156 & -0.0626 & 3.22 & -7.64 & -0.657 & -0.908 & 1100 \\
\hline *SA & -5.74 & 0.149 & 1.08 & -0.591 & 0.263 & 0.912 & 2.07 & $5.54 \mathrm{e}+06$ \\
\hline$* \mathrm{SM}$ & 656 & 1.88 & 1.6 & 2.45 & -841 & -0.0146 & 5.59 & 1610 \\
\hline *ST & 1.51 & 0.956 & 1.45 & -1.06 & 1.45 & 0.85 & -1.5 & 4250 \\
\hline$* \mathrm{VE}$ & -22 & 32.6 & 0.272 & 5.35 & 2.37 & -1.03 & -1.98 & 820 \\
\hline *VI & -4180 & -4170 & -0.00772 & 3.17 & 1.61 & 0.451 & 2.73 & $9.64 e+06$ \\
\hline
\end{tabular}

The muscle abbreviations are $\mathrm{AD}=$ Adductor Magnus, $\mathrm{ADl}=$ Adductor Longus, $\mathrm{BI}=\mathrm{Biceps}$ Femoris, GA=Gastrocnemius, $\mathrm{IP}=\mathrm{Ilio}-\mathrm{Ps}$ soas, $\mathrm{PE}=$ Peroneus, $\mathrm{PT}=$ Pectineus, $\mathrm{QF}=$ Quadratus Femoris, RA=Rectus Anticus, $\mathrm{RI}=$ Rectus Internus, RIm=Rectus Internus Minor, $\mathrm{SA}=\mathrm{Sartorius,}$ $\mathrm{SM}=$ Semimembranosus, $\mathrm{ST}=$ Semitendinosus, $\mathrm{VE}=$ Vastus Externus, and VI=Vastus Internus. The subscripts " $\mathrm{k}$ " and " $\mathrm{h}$ " indicate parameters of the knee and hip sinusoids respectively. The 12 muscles marked with asterisks (*) were typically implanted with EMG electrodes in our spinal stimulation experiments (Bizzi et al., 1991). The variables' units are millimeters $\left(L 0, A_{\mathrm{k}}, A_{\mathrm{h}}\right)$, radiants- 1 (freq $\mathrm{k}_{\mathrm{k}}$, freq $\mathrm{q}_{\mathrm{h}}$ ), radians $\left(\mathrm{phi}_{\mathrm{k}}\right.$, phi $\left.\mathrm{h}_{\mathrm{h}}\right)$ and $0.00125 \mathrm{~N}$ (scale). The large scale values in some of the models occur because this models yields good estimates of length changes, but it is not calibrated for absolute muscle lengths.

(force times displacement) with respect to coordinate transformations:

$$
\begin{aligned}
\text { muscle work } & =\text { tension } \Delta \text { length }=\text { skeletal work } \\
& =\operatorname{torque}^{T} \delta \theta
\end{aligned}
$$

where $\Delta$ length is an infinitesimal change in muscle length, $\delta \theta$ is an infinitesimal change in the vector of joint angles, and torque ${ }^{T}$ is the transpose of the vector of joint torques. Muscle tension is the linear force exerted by the muscle. ${ }^{5}$

The coordinate transformation for a muscle is the function, length $(\theta)$, that transforms the joint coordinate system of joint angles, $\theta$, into the muscle coordinate system of muscle lengths. Rearranging Equation 1, we have an equation for the torque vector produced by a single muscle:

$$
\text { torque }=\text { tension } *(\Delta \text { length }(\theta) / \delta \theta)^{T}
$$

We used the relation in Equation (2) to convert our muscle stimulation data into muscle models. Its second term, $(\Delta \text { length }(\theta) / \delta \theta)^{\mathrm{T}}$, is a vector of derivatives. Thus, we can compute the right side of the equation from a simple length function, provided the muscle tension is a simple function of muscle length and provided that we can compute the two required derivatives of the length function. There are many different length functions for which this is possible, and we used sinusoids (Lieber \& Boakes, 1988; Lieber \& Shoemaker, 1992) as follows:

$$
\begin{aligned}
& \text { length(hip angle, knee angle) } \\
& =L 0+A_{\mathrm{h}} * \cos \left(\text { freq }_{\mathrm{h}} * \text { hip }+\mathrm{phi}_{\mathrm{h}}\right)+A_{\mathrm{k}} \\
& \quad * \cos \left(\text { freq }_{\mathrm{k}} * \text { knee }^{2} \text { phi }_{\mathrm{k}}\right)
\end{aligned}
$$

There are seven external parameters in our length function: $L 0, A_{\mathrm{h}}$, freq $\mathrm{h}_{\mathrm{h}}, \mathrm{phi}_{\mathrm{h}}, A_{\mathrm{k}}$, freq $\mathrm{q}_{\mathrm{k}}$, and $\mathrm{phi}_{\mathrm{k}}$. The parameters with the "h" subscript refer to the part of the muscle-length function that depends on the hip angle. The parameters with the " $k$ " subscript refer to the part of the muscle-length function that depends on the knee angle. Note that the derivatives of this length function with respect to hip angle and knee angle are both easy to determine.

For the length-tension function, we used a sigmoid:

tension(hip, knee) $=$ scale * sigmoid(length(hip, knee)) 
Thus, our tension values ranged between 0 and scale (the eighth parameter in the model). The sigmoid partially approximates the shape of known lengthtension properties of muscles (Rack \& Westbury, 1969).

In order to fix the parameter values for a muscle, we use the errors in the equations

$$
\text { torque }=\text { tension } * \Delta \text { length } / \delta \theta
$$

to update the eight parameters in the model. The left side of the equation consisted of our many torque measurements for the muscle in question. The right side of the equation was determined by our eight parameter values and the (hip, knee) coordinates of the corresponding torque measurements. The parameter values were adjusted so as to minimize the sum of squared errors between all the measured and estimated torques for the muscle. The results of these least squares approximations for 16 muscles in the frog's hindlimb are listed in Table 2 . The force field correlations between the models and the measured force fields for all the muscles are summarized in a histogram in Figure 7.

\section{Testing the Accuracy of the Model}

We used our muscle models and recorded EMGs to predict the force vectors that should be observed at the ankle. The EMGs were recorded during microstimulation of the spinal cord and were paired with the force vectors at the ankle that were recorded at the same time. Thus, we were able to use the EMGs and the muscle models to predict the forces that we had already measured, and thereby test the accuracy of the muscle models.

Figure 14 shows the steps involved in predicting force vectors from a collection of EMG signals. Force prediction involves multiplying each muscle's model-predicted force vector by its respective EMG signals. We then added up all the individual muscle*EMG forces (see Hof \& Van Den Berg, 1977):

$$
\begin{gathered}
\text { force(frog, } \mathrm{h}, \mathrm{k}, \mathrm{t})=\Sigma_{\text {muscles }} \\
M(\text { muscle }, \mathrm{h}, \mathrm{k}) * \text { gain(frog, muscle) } \\
* \text { EMG }(\text { frog, muscle, } \mathrm{h}, \mathrm{k}, \mathrm{t})
\end{gathered}
$$

In Equation (3), the muscle model, $M$, indicates an estimate of the force generated by each muscle at a specific (h, k) configuration of hip and knee angles. These static force fields are each multiplied by a gain factor, which is specific to each muscle of each frog, and by the measured EMG signal, which is specific to the frog, the muscle, the posture, and varies over time. These weighted-model terms (the second line of the equation) are added together to give an estimated force vector signal for the particular EMG electrode placement and the limb position at which the EMG data was gathered. Note that in the right-hand side of Equation (3), the only term that depends explicitly on time is the
EMG. Thus, at each location $(x, y)$, a single value of gain and of the model (muscle) is used to account for the entire time history of the force. The predictions are also necessarily dependent on the frog-specific gain factors, which represent the variability in the conditions under which the EMG signals were recorded.

\section{Computing Gain Factors}

We lumped the several factors that affect the translation from an EMG signal to a muscle force-different electrode transduction efficacies, different balances of muscle fiber types, and so forth-into a single EMG-to-force gain factor for each muscle of each frog. For each frog, we used least-squares estimation to find the best collection of gain factors. The gain factors were also constrained to be positive and not too large (Cholewicki \& McGill, 1994): The ratio of the maximum gain factor to the minimum gain factor could not exceed a value of 20 . The gain factors were computed using the operations shown in Figure 14. The gains were those for which we found the best match between the predicted and observed forces at the ankle. We performed these computations for each animal on a randomly chosen subset of trials in its data set. We then removed these "training" trials from the data set for the purpose of subsequent statistical tests.

\section{Statistical Tests of Predicted Endpoint Force}

We used the angle between the actual force vector, $a\left(t_{\mathrm{k}}\right)$, recorded at the ankle, and the predicted force vector, $p\left(t_{\mathrm{k}}\right)$, at a single common time index, $t_{\mathrm{k}}$, to quantify goodness-of-fit. We chose the time index by selecting the best correlated pair, $a\left(t_{\mathrm{k}}\right)$ and $p\left(t_{\mathrm{k}}\right)$, within $100 \mathrm{msec}$ of the time index of the peak in actual force magnitude. For example, to compare the traces in Figure 14, we computed the correlations of $a\left(t_{\mathrm{k}}\right)$ and $p\left(t_{\mathrm{k}}\right)$ over indexes $t_{\max } \pm 100 \mathrm{msec}$. The time, $t_{\text {max }}$, of the actual peak force magnitude in the data used to construct Figure 14, is $550 \mathrm{msec}$. The best correlated pair over $t_{\max } \pm 100$ msec occurs at $t_{\mathrm{k}}=460$ $\left(t_{\max }-90 \mathrm{msec}\right)$, so the orientations of $a(460)$ and $p(460)$ were used to make one data point in Figure 9 out of the data trace and prediction trace shown in Figure 14 .

\section{Using Force Field Muscle Models to Examine Muscle Combinations}

We used our force field muscle models to learn about the range of possible workspace locations at which each muscle combination could stabilize the limb. A muscle combination is represented as a binary vector. Each muscle is either contained in the combination (1) or not (0). With 16 modeled muscles, there are $2^{16}=65,536$ possible muscle com- 


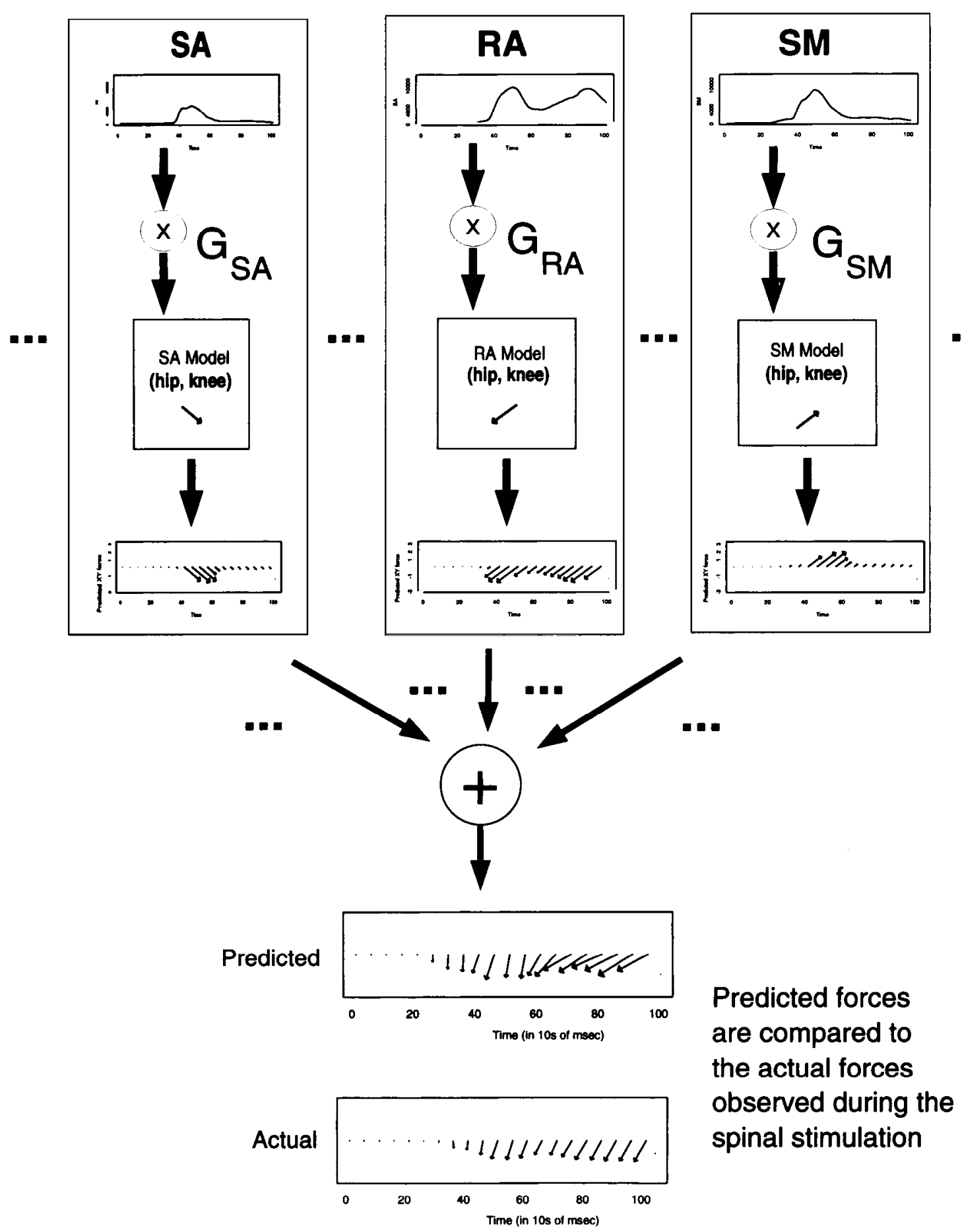

Figure 14. Reconstructing forces from EMGs: The three boxes in the top part of the figure show for three muscles the steps that we performed for all of the muscles in order to construct the predicted force trace at the bottom of the figure. The rectified and filtered EMG signals for Sartorius (SA), Rectus Anticus (RA), and Semimembranosus (SM) are shown in the top part of the boxes. We first multiply each filtered EMG signal by its empirical gain factor, $G$ (see text). The weighted EMG signal serves as time-varying scale factor for the force vector of its corresponding muscle model. The muscle models each take the hip angle and knee angle as inputs, and return a force vector. The product of the gain, the EMG, and the model force vector is shown at the bottom of each box. Finally, the force vectors are added together to produce the predicted forces at the bottom of the figure. The actual force trace observed with these EMG signals is also shown for comparison. 
binations. For each of these combinations, there are infinitely many possible real-valued activation patterns. For each combination, we generated 50-8500 random activation patterns. ${ }^{6}$ The number of random activation patterns we generated for a given muscle combination did not directly depend on the number of muscles in it.

Each random activation pattern produced a force field which was the sum of model force fields weighted by random values. From these force fields, several different aspects of the mechanical action of a muscle synergy could be examined. In this study, we focused on the muscle synergy's stability properties. We define stability by stable positions of the ankle. Namely, a CEP is a limb configuration at which (1) the torque magnitude is zero, and (2) any deviation away from the CEP results in torques pushing the limb back to the CEP.

For each random muscle activation pattern, we examined whether the resulting force field contained a stable limb configuration, a CEP, within the animal's workspace (hip $>-110^{\circ}$ and $<80^{\circ}$; knee $>10^{\circ}$ and $<170^{\circ}$ for a total
Figure 15. Histograms of two properties of muscle combinations: (A) Histogram of reliability values. Each computed force field either pointed to a CEP within the workspace or it did not. The reliability of a muscle combination is the proportion of its tested random activation patterns that result in a CEP. (B) Histogram of localization values. The localization value for a muscle combination is the maximal area of the distribution of CEP locations in the workspace. Muscle combinations that produced no CEPs (that is, reliability $=0$ ) have localization values of 0 . Otherwise, localization is the product of the spread in hip angle values and knee angle values at the computed equilibrium points.

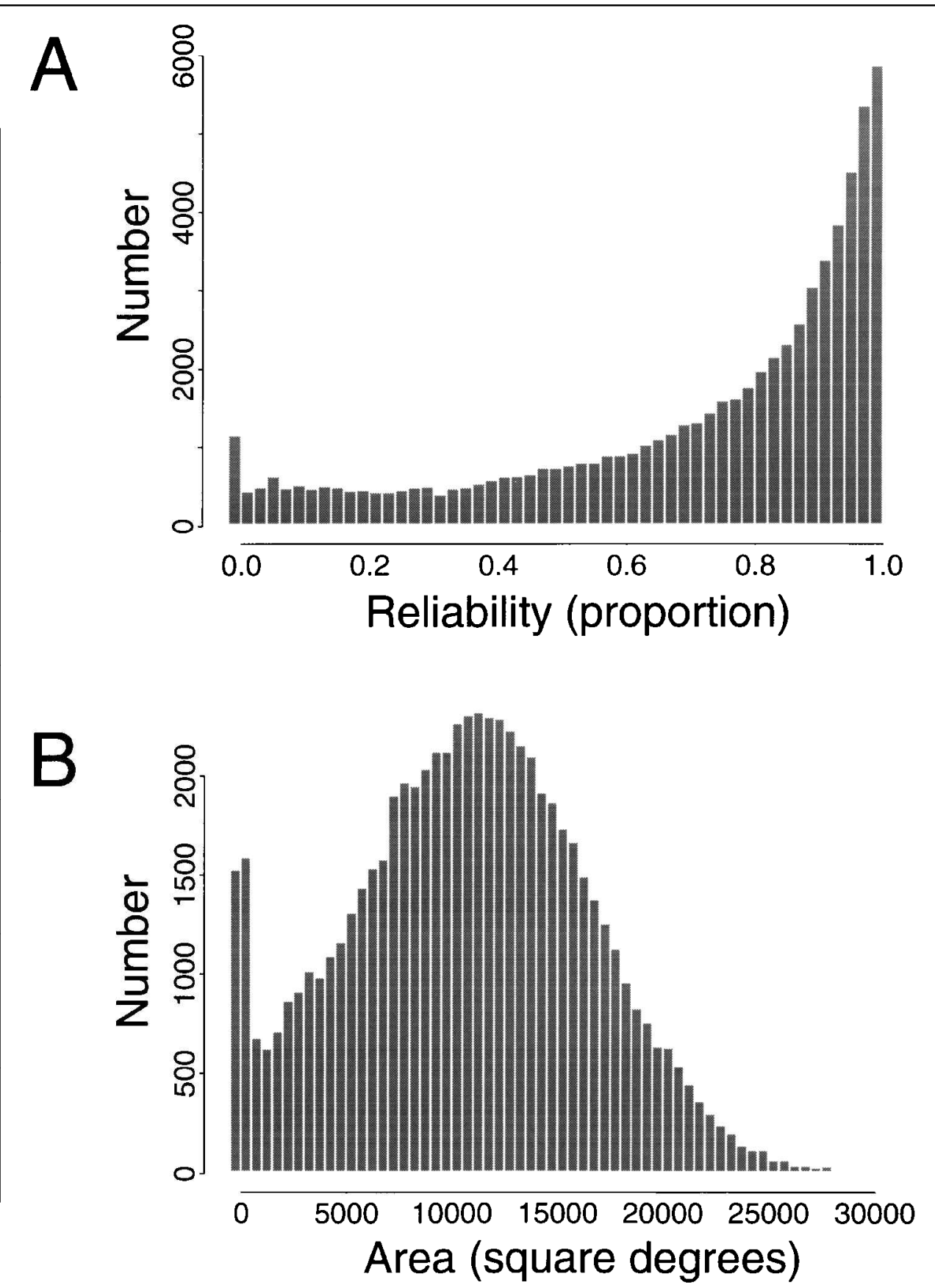


maximal workspace area of 30,400 square degrees). If the activation pattern did produce a CEP, the location of the sable position was recorded.

Our procedure consists of an "Outer Loop", which is performed $2^{16}$ times (once for each muscle combination), and an "Inner Loop" which is performed 50-8500 times for a given muscle combination, $\mathrm{C}$ :

Outer Loop: For each muscle combination, C

\section{Perform Inner Loop to estimate CEP distribution} of $\mathrm{C}$

2. (Figure 10B) Generate a random activation value for each muscle in $\mathrm{C}$

3. Weigh each muscle model by its random activation value

4. Sum the weighted muscle model force fields to produce a model of the total force field

5. Does it converge? Where? (Figure 10C)

6. Store the answers and return to step 1.

7. Compute and store the $(x, y)$ location of the joint space centroid of the CEP distribution of $\mathrm{C}$

As a result of storing all the CEP locations for each possible muscle combination, we were able to examine the properties of equilibrium point distributions. We were particularly interested in two properties of the distributions: "reliability" and "localization". The reliability of a muscle combination is the proportion of all the tested random activation patterns that produced a CEP. The localization of a muscle combination is the maximal two-dimensional area of the distribution of CEP locations (in joint space) This value is the product ( $\max$ knee- min knee) and (max hip- min hip).

Figure 15 shows the histograms of the reliability and localization values for all 65,536 possible combinations of 16 muscles. Note in Figure 15A that some muscle combinations have high reliability values - that is, they frequently produced convergent force fields. For example, Figure 15A shows that approximately 1000 muscle combinations had $60 \%$ reliability. For these 1000 different muscle combinations, 6 of every 10 random activation patterns resulted in a convergent force field. Some muscle combinations had small localization values (Figure 15B). The CEPs of these combinations fell in roughly the same place in the workspace. For example, Figure 15B shows that approximately 2000 different muscle combinations had a localization value of 15,000 square degrees, which is about half the workspace. For those 2000 different muscle combinations, all of the convergent force fields produced by random activation had CEPs that fell within some 15,000 square degree (joint space) box in the workspace. We called the muscle combinations that were both localized and reliable "robust" because these combinations could, apparently, produce a CEP in approximately the same workspace location, even though there is activation noise in the nerves and muscles.

\section{Acknowledgments}

This work was supported by NIH Grants NS09343 and MH48185 and ONR Grant N00014/88/K0372. We are also indebted to the thoughtful insights of Andrea D'Avella, James Galagan, Dr. Francesca Gandolfo, Dr. Terry Sanger, Dr. Judith Schotland, and Dr. Mathiew Tresch.

\section{Notes}

1. To understand the problem of degrees of freedom, touch the tip of your nose while moving your elbow to all the different positions it can move to. There are arbitrarily many different places your elbow can be while still allowing your finger to rest on the tip of your nose. This is an example of extra degrees of freedom: how and why is a particular elbow location used? There are many equally competent possibilities available to the CNS. The degrees of freedom problem is still greater in the case of the selection by the CNS of a set of muscle activations to achieve a movement, because there is a still greater excess of options in which muscles fibers to activate, for how long, and starting when.

2 . The displayed groupings are for convenience only. The force fields described here have only been analyzed in the horizontal plane. The actual force fields generated by the hindlimb muscles are (at least) 3-D entities. The degree of similarity of the models in the horizontal plane may not correspond to an equivalent similarity in three dimensions. 3. Muscles EC, TP, and TA are also all knee extensor muscles. We have removed these muscles from further consideration, as they were generally too small and weak to permit reliable isolation for the stimulation experiments. Muscle Pyriformis (PY) is an easily isolated Hip Extensor muscle, but due to its weakness of action in the $(x, y)$ plane we removed it from the simulations.

4. We have found that the latency to peak force magnitude is nearly constant across the workspace. Isometric force fields produced by sampling the data at a single latency applied across the workspace (for example, $\operatorname{FF} 0(x, y)=\mathrm{FF}(x, y, t 0)$ ) are similar in most cases to those achieved with the peak-force method described here. Thus, a time-varying force field, $\operatorname{FF}(x$, $y, t)$, may be decomposed as $\operatorname{FFi}(x, y)^{*} G(t)$, for some magnitude function, $G(t)$.

5. We use "tension" incorrectly here to refer to linear force, rather than linear force per unit cross-sectional area, because we wish to avoid a confusion of terms between endpoint force and linear force.

6. For each boolean combination of muscles in the $2^{16}$ possible combinations, we initially generated 50 random activation patterns. If this random sampling produced two or more convergent fields (which is defined below as $4 \%$ reliability or greater) the combination was explored with 500 more random patterns. In addition to this systematic examination, we also explored a smaller set of boolean combinations with 8500 samples each. These explorations are the subject of Figure 12.

\section{REFERENCES}

Bernstein, N. (1971). Biodynamics of locomotion. Reprinted in human motor actions: Bernstein revisited. In T. A. Whiting (Ed.), Advanced Psychology, 17, 171-222. Amsterdam: Elsevier.

Bizzi, E., Mussa-Ivaldi, F. A., \& Giszter, S. F. (1991). Computations underlying the execution of movement: a novel biological perspective. Science, 253, 287-291.

Buchanan, T. S., Almdale, D. P., Lewis, J. L., \& Rymer, W. Z. (1986). Characteristics of synergic relations during isometric 
contractions of human elbow muscles. Journal of Neurophysiology, 56, 1225-1241.

Cholewicki, J., \& McGill, S. M. (1994). EMG assisted optimization: A hybrid approach f or estimating muscle forces in an indeterminate biomechanical model. Journal of Biomechanics, 27, 1287-1289.

Crago, P. E., Peckham, P. H., \& Thrope, G. B. (1980). Modulation of muscle force by recruitment during intramuscular stimulation. IEEE Transactions on Biomedical Engineering BME, 27, 679-684.

Efron, B., \& Tibshirani (1982). The Jackknife, the Bootstrap, and other resampling plans. Society for Industrial and Applied Mathematics.

Giszter, S. F., Mussa-Ivaldi, F. A., \& Bizzi, E. (1993). Convergent force fields organized in the frog spinal cord. Journal of Neuroscience, 13, 467-491.

Hof, A. L., \& Van Den Berg, J. W. (1977). Linearity between the weighted sum of the EMGs of the human triceps surae and the total torque. Journal of Biomechanics, 10, 529-539.

Jacobs, R. A., \& Jordan, M. I. (1993). Learning Piecewise Control Strategies in a Modular Neural Network Architecture. IEEE Transactions on Systems, Man, and Cybernetics, 23, 337345.

Lan, N., \& Crago, P. E. (1992). A noninvasive technique for in vivo measurement of joint torques of biarticular muscles. Journal of Biomechanics, 25, 1075-1079.

Lieber, R. L., \& Boakes, J. L. (1988). Muscle force and moment arm contributions to torque production in frog hind limb. American Journal of Physiology, 254, C769-C772.

Lieber, R. L., \& Shoemaker, S. D. (1992). Muscle, joint, and tendon contributions to torque profile in frog hip-joint. American Journal of Physiology, 263, R586-R590.

Loeb, E. P., Giszter, S. F., Borghesani, P., \& Bizzi, E. (1993). Effects of dorsal root cut on the forces evoked by spinal microstimulation in the spinalized frog. Somatosensory and Motor Research, 10, 81-95.

Loeb, G. E., He, J., \& Levine, W. S. (1989). Spinal cord circuits:
Are they mirrors of musculoskeletal mechanics? Journal of Motor Behavior, 21, 473-491.

MacPherson, J. M. (1991). In D. R. Humphrey and H. J. Freund (Eds.), How flexible are muscle synergies? Motor control concepts and issues.

Mussa-Ivaldi, F. A. (1992). From basis functions to basis fields: Vector-field approximation from sparse data. Biological Cybernetics, 67, 479-489.

Mussa-Ivaldi, F. A., Giszter, S. F., \& Bizzi, E. (1994). Linear combinations of primitives in vertebrate motor control. Journal of Neurobiology, 91, 7534-7538.

Rack, P. M. H., \& Westbury, D. R. (1969). The effects of length and stimulus rate on tension in the isometric cat soleus muscle. Journal of Physiology (London), 240, 443-460.

Saltiel, P., Tresch, M. C., \& Bizzi, E. (1998). Spinal cord modular organization and rhythm generation: An NMDA iontophoretic study in the frog. Journal of Neurophysiology, 80, 2323-2339.

Sherington, C. (1961). The Integrative action of the nervous system, 2nd Ed., New Haven: Yale University Press.

Soechting, J. F., \& Lacquaniti, F. (1989). An assessment of the existence of muscle synergies during load perterbations and intentional movements of the human arm. Experimental Brain Research, 74, 535-548.

Wickland, C. R., Baker, J. F., \& Peterson, B. W. (1991). Torque vectors of neck muscles in the cat. Experimental Brain Research, 84, 649-659.

Winters, J. M., \& Stark, L. (1988). Estimated mechanical properties of synergistic muscles involved in movements of a variety of human joints. Journal of Biomechanics, 21, 10271041.

Zajac, F. E., \& Winters, J. M. (1990). Modeling musculoskeletal movement systems: Joint and body segmental dynamics, musculoskeletal actuation, and neuromuscular control. In J. M. Winters \& S. L.-Y. Woo (Eds.), Multiple muscle systems: Biomechanics and movement (p. 121-147). 